\title{
Combinatorial approach to Mathieu and Lamé equations
}

Cite as: J. Math. Phys. 56, 072302 (2015); https://doi.org/10.1063/1.4926954

Submitted: 09 April 2015 . Accepted: 30 June 2015. Published Online: 21 July 2015

Wei He

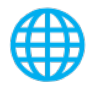

\section{ARTICLES YOU MAY BE INTERESTED IN}

Deformed Calogero-Sutherland model and fractional quantum Hall effect Journal of Mathematical Physics 58, 011902 (2017); https:// doi.org/10.1063/1.4973509

Whittaker-Hill equation and semifinite-gap Schrödinger operators Journal of Mathematical Physics 51, 072108 (2010); https:// doi.org/10.1063/1.3455367

Analytic representations with theta functions for systems on $\mathbb{Z}(\mathrm{d})$ and on \#\# Journal of Mathematical Physics 56, 072108 (2015); https:// doi.org/10.1063/1.4927256

Where in the world is AIP Publishing? Find out where we are exhibiting next 


\title{
Combinatorial approach to Mathieu and Lamé equations
}

\author{
Wei He ${ }^{\mathrm{a})}$ \\ Center of Mathematical Sciences, Zhejiang University, Hangzhou 310027, China and \\ Instituto de Física Teórica, Universidade Estadual Paulista, Barra Funda 01140-070, \\ São Paulo, SP, Brazil
}

(Received 9 April 2015; accepted 30 June 2015; published online 21 July 2015)

\begin{abstract}
Based on some recent progress on a relation between four dimensional super YangMills gauge theory and quantum integrable system, we study the asymptotic spectrum of the quantum mechanical problems described by the Mathieu equation and the Lamé equation. The large momentum asymptotic expansion of the eigenvalue is related to the instanton partition function of supersymmetric gauge theories which can be evaluated by a combinatorial method. The electro-magnetic duality of gauge theory indicates that in the parameter space, there are three asymptotic expansions for the eigenvalue, and we confirm this fact by performing the Wentzel-Kramers-Brillouin (WKB) analysis in each asymptotic expansion region. The results presented here give some new perspective on the Floquet theory about periodic differential equation. @ 2015 AIP Publishing LLC. [http://dx.doi.org/10.1063/1.4926954]
\end{abstract}

\section{INTRODUCTION}

Nonrelativistic quantum mechanics is naturally related to the second order differential equations. Exactly solvable quantum mechanics models play a very fundamental role in demonstrating the basic concepts and methods of quantum theory. A special interesting class of ordinary differential equations are those with periodic coefficients; in quantum mechanics, they describe a particle moving in one dimensional periodic potentials. Therefore, spectral problem of these equations is of particular interesting for both quantum physics and functional analysis. In this paper, we study two typical equations of this kind, the Mathieu equation and the Lamé equation. They are respectively associated to two mathematicians who studied them in the 19th century. ${ }^{1,2}$ The two equations and their solutions are related to each other: the Mathieu equation is a particular limit of the Lamé equation.

Our approach to the problem would be different from the conventional methods; the spectral solution of the equations is studied in the context of their relation to supersymmetric quantum gauge theory. It is surprising that such simple quantum mechanics problems are related to seemly much more complicated quantum gauge theories. In general, the quantum dynamics of gauge theory is very complicated; nevertheless, in supersymmetric gauge theory models, the dynamics is tamed and many exact results are obtained. The quantum gauge theory relevant to our study is the $\mathrm{N}=2$ Seiberg-Witten gauge theory ${ }^{3,4}$ subject to the $\Omega$ background deformation. ${ }^{22}$ According to the recent proposal of Nekrasov and Shatashvili, ${ }^{5-7}$ the low energy dynamics of the deformed $\mathrm{N}=2$ gauge theories is equivalent to the spectral problem of some quantum integrable systems. This connection leads to two particular examples relating quantum gauge theory to the periodic Schrödinger operator: the $\mathrm{SU}(2)$ pure gauge theory is related to the Mathieu equation and the $\mathrm{SU}(2)$ gauge theory with an adjoint matter (the $\mathrm{N}=2^{*}$ theory) is related to the Lamé equation.

We summarise the structure of the paper as follows. In Section II, we explain the relation between differential equations and corresponding gauge theories. In Section III, we show how the asymptotic spectrum of the Mathieu equation is related to the effective action of SU(2) pure gauge

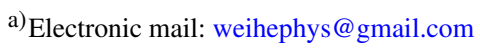


theory. In Section IV, we obtain the large quasimomentum asymptotic eigenvalue for the Lamé equation from the instanton partition function of the $\mathrm{N}=2^{*}$ gauge theory. In Section $\mathrm{V}$, we perform the Wentzel-Kramers-Brillouin (WKB) analysis for the large quasimomentum eigenvalue, based on the Floquet theory, to confirm the results obtained from the gauge theory. In Section VI, using the same differential operators obtained in Sec. V, we obtain other two eigenvalue expansions; the relation between the three eigenvalue expansions can be understood by the electro-magnetic duality of the effective gauge theory. Section VII is for conclusion and open problems. Some technical details are presented in the Appendixes.

\section{DIFFERENTIAL EQUATIONS AND GAUGE THEORY}

In this section, we first explain some basic facts about the Mathieu and the Lamé equations, in the context of Floquet theory. We are interested in their asymptotic energy spectrum which by itself is an interesting topic in quantum theory. Then, we explain how the spectral theory of Schrödinger operators is related to the instanton partition function of gauge theories.

\section{A. The Mathieu equation}

The Mathieu differential equation is

$$
\frac{d^{2} \Psi}{d z^{2}}+(\lambda-2 h \cos 2 z) \Psi=0
$$

The related modified Mathieu equation is obtained by $z \rightarrow i z$, then cosine potential is transformed to the hyperbolic potential. The (modified) Mathieu equation is useful in various mathematics and physics problems, including the separation of variables for the wave equation in the elliptical coordinates, describing a quantum particle moving in periodic potential. It also appears in problems such as wave scattering by D-brane of string theory ${ }^{8}$ and reheating process in some inflation models. ${ }^{9}$ In quantum integrable theory, it is the two body Shrödinger equation of the Toda system.

The potential $\cos 2 z$ is periodic along the real axes; therefore, the Mathieu equation is a Floquet differential equation. The Floquet (or Floquet-Bloch) solution is a function with the following monodromy property under the shift of argument by a period $\pi$ :

$$
\Psi(z+\pi)=e^{i \pi v} \Psi(z),
$$

where the quantity $v=v(\lambda, h)$ is the Floquet exponent. For periodic Shrödinger equation, the Floquet exponent of wave function is the quasimomenta of particles. Our focus in this paper is the asymptotic solution for the eigenvalue $\lambda$ as a function of $v, h$.

In general, the eigenvalue relation $\lambda(v, h)$ cannot be written in elementary functions, and only asymptotic expansions are obtained when a small expansion parameter is available. For the Mathieu equation, there are three asymptotic expansions for $\lambda$, and the leading order terms are

$$
\begin{aligned}
& \lambda \sim v^{2}+O\left(\frac{h^{2}}{v^{2}}\right), \quad v \gg 1, \frac{h}{v^{2}} \ll 1, \\
& \lambda \sim \pm 2 h+O(v \sqrt{h}), \quad \frac{h}{v^{2}} \gg 1 .
\end{aligned}
$$

These are well known results about the Mathieu equation; they can be found in handbooks such as Refs. 10-12. We would explain their relation to gauge theory effective action in Sec. III.

\section{B. The Lamé equation}

The Lamé differential equation contains an elliptic potential, and it can be written in several forms. In the Jacobian form, it is

$$
\frac{d^{2} \Phi}{d \varkappa^{2}}-\left[A+n(n-1) k^{2} \operatorname{sn}^{2} \varkappa\right] \Phi=0
$$


where $\operatorname{sn} \varkappa=\operatorname{sn}\left(\varkappa \mid k^{2}\right)$ is the Jacobi elliptic function. In the Weierstrass form, it is

$$
\frac{d^{2} \Phi}{d z^{2}}-[B+n(n-1) \wp(z)] \Phi=0,
$$

where $\wp(z)=\wp\left(z ; \omega_{1}, \omega_{2}\right)$ is the Weierstrass elliptic function. The coefficient $n(n-1)$ is in accordance with the usual literature, but we will not discuss whether $n$ is an integer or not which is crucial for the classification of the solution; this does not affect the discussion of asymptotic spectrum. The Lamé equation is obtained from separation of variables for the Laplace equation in the ellipsoidal coordinates. In quantum physics, it is the Shrödinger equation of two body elliptic Calogero-Moser system.

The elliptic modulus of the function sn $\varkappa$ is $k$, while the elliptic modulus of the function $\wp(z)$ is $q=\exp \left(i 2 \pi \frac{\omega_{2}}{\omega_{1}}\right)$; they are related through relation (A5) given in Appendix A. Equations (4) and (5) are related by a change of variables, ${ }^{13}$

$$
\frac{x-i K^{\prime}}{\sqrt{e_{1}-e_{2}}}=z
$$

where $K^{\prime}=K\left(k^{\prime}\right)$ is the complete elliptic integrals of the first kind, $k^{\prime}=\sqrt{1-k^{2}}$ is the complementary module, and $e_{i}(q)=\wp\left(\omega_{i}\right)$. Their eigenvalues are related by

$$
B=\left(e_{1}-e_{2}\right) A-e_{2} n(n-1)
$$

or

$$
A=\frac{B}{e_{1}-e_{2}}-\frac{1+k^{2}}{3} n(n-1) .
$$

The two equations are equivalent provided the change of variables is smooth. However, the change is actually singular in the limit $k \rightarrow 0$, as in this limit $K^{\prime} \rightarrow \infty$. Therefore, the two equations may reduce to different equations in limits involving $k \rightarrow 0$.

The elliptic functions $\operatorname{sn} \varkappa$ and $\wp(z)$ are doubly periodic. The periods of $\operatorname{sn} \varkappa$ are $4 K$ and $2 i K^{\prime}$ and the periods of $\wp(z)$ are $2 \omega_{1}$ and $2 \omega_{2}$. They are related by

$$
\omega_{1}=\frac{K}{\sqrt{e_{1}-e_{2}}}, \quad \omega_{2}=\frac{i K^{\prime}}{\sqrt{e_{1}-e_{2}}} .
$$

Since we have $\operatorname{sn}(\varkappa+2 K)=-\operatorname{sn}(\varkappa), \operatorname{sn}\left(\varkappa+i K^{\prime}\right)=\frac{1}{k \operatorname{sn}(\varkappa)}$, the periods of the potential $\operatorname{sn}^{2} \varkappa$ are $2 K$ and $2 i K^{\prime}$.

The Lamé equation also falls into the equations of Floquet type; however, as the potential is the elliptic function, a generalised version of the Floquet theory is needed. The generalised Floquet theory for elliptic potential is one of the main results of this paper, and the details are given in Secs. III-VII. One of the periods $2 K$ (or $2 \omega_{1}$ ) actually can be treated by the conventional Floquet theory, see Section $\mathrm{V}$ for detail. When the coordinate $\varkappa$ shifts a period $2 K$, or coordinate $z$ shifts a period $2 \omega_{1}$, the phase shift of the function $\Phi$ defines the Floquet exponent by

$$
\Phi(\varkappa+2 K)=e^{i 2 K \mu} \Phi(\varkappa)
$$

or

$$
\Phi\left(z+2 \omega_{1}\right)=e^{i 2 \omega_{1} v} \Phi(z) .
$$

We use different letters $\mu$ and $v$ to denote the exponent for the Lamé equation, depending on its appearance in solution of the equation in Jacobian form (4) or in Weierstrass form (5). The phase shifts should be the same, then we have the relation

$$
\frac{v}{\mu}=\frac{K}{\omega_{1}}=\sqrt{e_{1}-e_{2}}
$$

In the limit $k \rightarrow 0, \mu$ and $v$ coincide. This limit is needed when we reduce the Lamé equation to the Mathieu equation, and indeed their eigenvalue expansions share some common features if we compare the formulae (19) and (34), (23) and (52). 
In the limit $k \rightarrow 0$, we have $2 K \rightarrow \pi$ and $2 i K^{\prime} \rightarrow i \infty^{\prime}$. This indicates the period $2 i K^{\prime}\left(\right.$ or $\left.2 \omega_{2}\right)$ is a bit different from the period $2 K$ (or $2 \omega_{1}$ ), and indeed it needs a special treatment when concerns the Floquet theory, see Section VI.

We would find the eigenvalue of Lamé equation, $A$ (or $B$ ), as a function of $\mu$ (or $v$ ) and $n, k$ (or $q$ ). The Lamé eigenvalue also has three asymptotic expansion regions, and their leading order terms are

$$
\begin{aligned}
& A \sim-\mu^{2}-\frac{\kappa^{2}}{2}+O\left(\frac{\kappa^{4}}{\mu^{2}}\right), \quad \mu \gg 1, \frac{\kappa}{\mu} \ll 1, \\
& A \sim-i 2 \kappa \mu+O\left(\mu^{2}\right), \quad \frac{\kappa}{\mu} \gg 1, \\
& A \sim-\kappa^{2}+i 2 \kappa \mu+O\left(\mu^{2}\right), \quad \frac{\kappa}{\mu} \gg 1,
\end{aligned}
$$

where $\kappa^{2}=n(n-1) k^{2}$. The more precise form of the expansions are in the formulae (36), (52), and (63). The first expansion was obtained recently by Langmann as a special case in his solution to quantum elliptic Calogero-Moser model, ${ }^{14}$ however expressed in terms of $B(v, n, q)$. The second expansion has been given by E. L. Ince, and later by Müller, see the book Ref. 12. To our knowledge, the third expansion is a new result not given in the previous literature. In the Secs. IV-VI, we derive all three asymptotic expansions in the context of the generalised version of Floquet theory.

In quantum mechanics we often study spectral solution with real eigenvalue; however, in this paper,we treat all quantities $z, \lambda, h$ and $\varkappa, A, n$ (and $z, B$ ) as complex variables. The $\mathrm{N}=2$ gauge theories are related to algebraic integrable systems where mechanical systems are investigated by complex analysis methods. The spectral curves of the integrable systems are defined over the field of complex numbers, the Abelian variety of the curve, which governs the linearized motion of corresponding mechanical system, is the Jacobian variety which carries a complex structure, i.e., a complex tori. Quantization of the algebraic integrable system needs to find the eigenvalues of complex Hamiltonian operators among which is the complex Schrödinger operator. When we compare the magnitude of these parameters with some numbers, we mean taking their absolute values or restoring their real values.

\section{The spectrum of Schrödinger operator and quantum gauge theory}

In this section, we explain the relation between integrable systems and quantum gauge theories, which provides the basic background for our study. It has been known that the exact solution of Seiberg-Witten on $\mathrm{N}=2$ supersymmetric Yang-Mills (SYM) gauge theory can be understood by the fact that the low energy dynamics on the special Kahler moduli space of gauge theory is equivalent to the dynamics of classical algebraic integrable system. ${ }^{16-21}$ Classical mechanical system can be quantised, and the quantization procedure can be understood as a deformation of the phase space and associated functional relation; correspondingly, the gauge theory also allows a deformation by background field parameterized by $\epsilon_{1}, \epsilon_{2},{ }^{22-26}$ and indeed the gauge theory deformation is exactly the quantization of classical mechanical system mentioned above. Their precise relation is recently pointed out by Nekrasov and Shatashvili: ${ }^{7}$ the Coulomb vacua of $\Omega$ deformed supersymmetric gauge theories, in the limit $\epsilon_{1}=\hbar, \epsilon_{2}=0$, are in one to one correspondence with the spectra of certain quantum integrable systems.

The action of the gauge theory has a scalar potential which leads to the vacuum expectation value (VEV) for the scalar; it breaks the gauge group and gives mass to some fields. The VEV takes value in the Cartan subalgebra of the gauge group, $\langle\phi\rangle=\sum_{i=1}^{r} \phi_{i} H_{i}$, with $r$ the rank of gauge group. The eigenvalues of the matrix $\langle\phi\rangle$ are denoted by $a_{i}$. The low energy effective theory is obtained by integrating all heavy particles and instanton effects, which is described by the prepotential function which includes a perturbative term and infinite many terms of instanton correction, $\mathcal{F}\left(a_{i}, q_{i n}\right)=\mathcal{F}^{\text {pert }}+\sum_{\ell=1}^{\infty} \mathcal{F}_{\ell} q_{i n}^{\ell}$, where $q_{i n}$ is the instanton expansion parameter. ${ }^{3,4}$ Upon the so called $\Omega$ background field deformation, the gauge theory remains well defined, and even better: while it is very difficult to compute the instanton contribution $\mathcal{F}_{\ell}\left(a_{i}, q_{i n}\right)$ in the undeformed gauge theory by quantum field theory method, the deformed prepotential $\mathcal{F}\left(a_{i}, q_{i n}, \epsilon_{1}, \epsilon_{2}\right)$ can be exactly 
computed by a localization method. ${ }^{22}$ In the limit $\epsilon_{2}=0$, the gauge theory is effectively confined on a two dimensional plane; therefore, the Gauge/Bethe correspondence of Refs. 5 and 6 relates the $\epsilon_{1}$ deformed gauge theory to quantum integrable system. More precisely, $\epsilon_{1}$ in the gauge theory plays the role of Planck constant, the $a_{i}$ are identified with the quasimomenta of excitations in integrable model, and the vacuum expectation value of gauge invariant operators $\left\langle\operatorname{Tr} \phi^{\ell}\right\rangle$ are identified with eigenvalues of the Hamiltonian operators $H_{\ell}$. Finally, the prepotential of gauge theory is identified with the Yang-Yang potential; the equation selecting the vacua of gauge theory is the Bethe equation selecting the allowed quasimomenta of integrable model.

In a few simple cases, this correspondence relates $\mathrm{SU}(2)$ gauge theories to 2-body quantum mechanics problems and provides simple examples to examine the relation in detail. The $\mathrm{SU}(2)$ pure gauge theory is related to the Schrödinger equation of 2-particle periodic Toda model, i.e., the Mathieu equation; SU(2) gauge theory with adjoint matter is related to the Schrödinger equation of 2-particle periodic elliptic Calogero-Moser model, i.e., the Lamé equation. ${ }^{7}$ The eigenvalues of Mathieu and Lamé equations are generally transcendental; in this paper, we are interested in their asymptotic solutions. Some results about their asymptotic solutions are already known, and here we revise the problem from the perspective of quantum gauge theory. Our focus is the asymptotic eigenvalue; we do not try to solve the asymptotic wave function, although it is argued to be related to gauge theory partition function in the presence of surface operator and can be computed by localization. ${ }^{27,28}$

For the $\mathrm{SU}(2)$ gauge theory, the only nontrivial $\operatorname{Tr} \phi^{\ell}$ is for $\ell=2$; it gives the eigenvalue for the Schrödinger equation. The expectation value $\left\langle\operatorname{Tr} \phi^{2}\right\rangle$ is a function of gauge theory parameters,

$$
\left\langle\operatorname{Tr} \phi^{2}\right\rangle=u\left(a, q_{i n}, \epsilon_{1}, \epsilon_{2}\right) .
$$

The Matone's relation ${ }^{29,30}$ states a relation between $u$ and the prepotential $\mathcal{F}$,

$$
2 u=q_{\text {in }} \frac{\partial}{\partial q_{\text {in }}} \mathcal{F} .
$$

As $u$ also expands according to the instanton parameter, $u=\sum_{\ell=0}^{\infty} u_{\ell} q_{i n}^{\ell}$, therefore we have

$$
2 u_{0}=q_{\text {in }} \frac{\partial}{\partial q_{\text {in }}} \mathcal{F}^{\text {pert }}, \quad 2 u_{\ell}=\ell \mathcal{F}_{\ell}, \quad \ell \geqslant 1 .
$$

This relation holds for generic deformation $\epsilon_{1,2}$, and after taking the limit $\epsilon_{2}=0$, we can interpret the relation in the context of quantum mechanical problem. As a consequence, if we properly identify a parameter in the quantum mechanical problem with the gauge theory parameter $q_{i n}$, then the $\ell$-th coefficients of $q_{i n}$-expansion of the eigenvalue is proportional to the $\ell$-th instanton contribution of $\mathrm{SU}(2)$ gauge theory prepotential.

\section{SPECTRUM OF THE MATHIEU EQUATION}

As the Mathieu and the Lamé equations are closely related to each other, the procedures of deriving their eigenvalues are largely parallel. The results in this section have already been given in earlier works. ${ }^{31-35}$ Here, we give a brief summary on the Mathieu eigenvalue as an educational example to illustrate, and to further explain, the basic method and logic of our approach.

There are three aspects we would explain. (1) One of the asymptotic eigenvalues, for which $v \gg 1$, is directly related the gauge theory partition function, in the way explained in Subsection II C. (2) An independent exact WKB analysis can be performed for the Schrödinger equation with periodic potentials to obtain the asymptotic eigenvalue for $v \gg 1$. During the process, we obtain a tower of higher order differential operators. (3) Last, the electro-magnetic duality of gauge theory indicates other two asymptotic expansions for the eigenvalue. Using the differential operators obtained before, we can compute the other two asymptotic expansions.

After understanding the procedure for the Mathieu eigenvalue, it is straightforward to apply this method to the Lamé eigenvalue problem. 


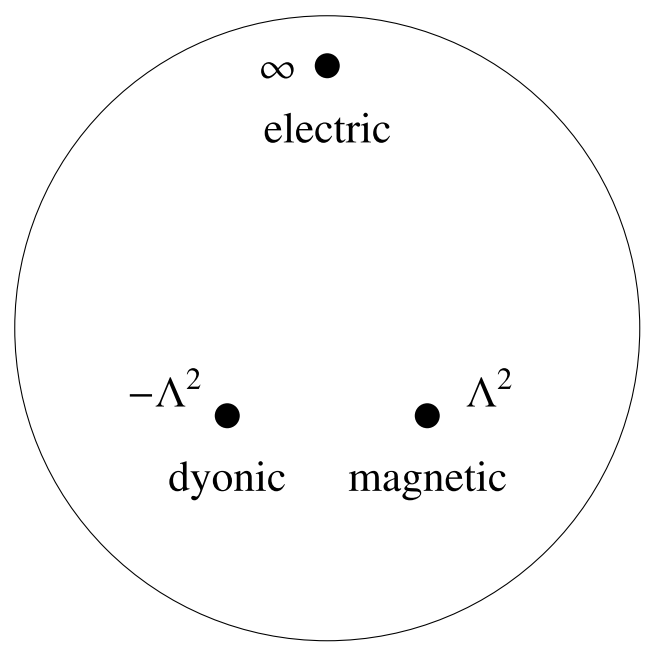

FIG. 1. Singularities in the $u$-plane.

\section{A. Combinatorial evaluating in the electric region}

First, we state the relation between singularities in the moduli space of the gauge theory and asymptotic expansion regions of the Mathieu eigenvalue. The moduli space of the $\mathrm{N}=2 \mathrm{SU}(2)$ pure gauge theory is parameterized by the only complex gauge invariant variable $u=\left\langle\operatorname{Tr} \phi^{2}\right\rangle$ which breaks SU(2) symmetry to Abelian $\mathrm{U}(1)$. The moduli space is singular at $u=\infty, \pm \Lambda^{2}$ in the sense that at each singularity, there are massless particles that can be used to describe the effective gauge theory as a weakly coupled theory. These singularities are labeled by the U(1) charges of the massless particles, denoted as "electric," "magnetic," and "dyonic," respectively. Near each singularity, the gauge theory has an unique weak coupling description; therefore, perturbative expansion is valid. Our calculation in Refs. 32 and 33 shows that there is a one to one correspondence between the perturbative expansion of gauge theory near a singularity in the moduli space and the asymptotic expansion for the corresponding Mathieu eigenvalue. In each asymptotic expansion region, the intervals between adjacent energy levels are very small compared to the eigenvalues themselves; therefore, the eigenstates are dense there, see Figs. 1 and 2.

The $\mathrm{N}=2$ gauge theory is formulated by a classical Lagrangian suitable for weak coupling region near $u \sim \infty$ where $\frac{\Lambda}{a} \ll 1$; the combinatorial method is used to compute the effective prepotential $\mathcal{F}\left(a, q_{i n}, \epsilon_{1}, \epsilon_{2}\right)$ at that point. ${ }^{22}$ Along with the logic explained in Sec. II, we identify the parameters of gauge theory and Mathieu eigenvalue as ${ }^{32}$

$$
\lambda=\frac{8 u}{\epsilon_{1}^{2}}, \quad v=\frac{2 a}{\epsilon_{1}}, \quad h=\frac{4 \Lambda^{2}}{\epsilon_{1}^{2}}=\frac{4 \sqrt{q_{i n}}}{\epsilon_{1}^{2}} .
$$

In order to compute formula (15), we need to compute the Nekrasov partition function from which the deformed prepotential $\mathcal{F}\left(a, q_{i n}, \epsilon_{1}, \epsilon_{2}\right)$ can be derived. ${ }^{22}$ There are formulae convenient for program treatment, ${ }^{35,36}$ and the complete expressions of the first three $\mathcal{F}_{1}, \mathcal{F}_{2}, \mathcal{F}_{3}$ are presented in

$$
\mathrm{V}(\mathrm{x})=\Lambda^{2} \cos 2 \mathrm{x} \equiv \text { electric } \infty
$$

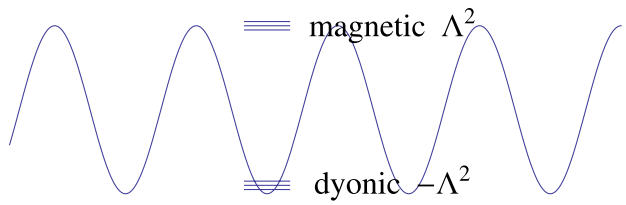

FIG. 2. Spectrum and duality of cosine-potential. 
Ref. 35. As observed in Ref. 34, according to Matone's relation (15), and taking into account (17), the Mathieu eigenvalue is

$$
\begin{aligned}
\lambda & =v^{2}+\sum_{\ell=1}^{\infty} \frac{\epsilon_{1}^{4 \ell-2}}{16^{\ell}} 4 \ell \mathcal{F}_{\ell}\left(2 a, \epsilon_{1}, \epsilon_{2}=0\right) h^{2 \ell} \\
& =v^{2}+\sum_{\ell=1}^{\infty} 4 \ell \mathcal{F}_{\ell}(v, 1,0)\left(\frac{h}{4}\right)^{2 \ell} .
\end{aligned}
$$

The leading term $v^{2}$ comes from the perturbative contribution. We have used the fact that the numerator and denominator of $\mathcal{F}_{\ell}\left(a, \epsilon_{1}\right)$ are homogeneous polynomials of $a, \epsilon_{1}$ of degrees $2 \ell+2$ and $6 \ell$, respectively. We get the asymptotic expansion

$$
\lambda=v^{2}+\frac{1}{2\left(v^{2}-1\right)} h^{2}+\frac{5 v^{2}+7}{32\left(v^{2}-1\right)^{3}\left(v^{2}-4\right)} h^{4}+\cdots,
$$

for $h \ll 1, v \gg 1$, and/or $h \gg 1, \frac{h}{v^{2}} \ll 1$.

\section{B. Extension to other regions}

A crucial ingredient of the Seiberg-Witten theory ${ }^{3}$ is that the effective gauge theory demonstrates electro-magnetic duality in the moduli space; the dual descriptions are valid at $u= \pm \Lambda^{2}$; accordingly, this indicates there are other two asymptotic expansions for the eigenvalue. In order to extend the asymptotic expansion from $u \sim \infty$ to other two regions near $u= \pm \Lambda^{2}$, we recall how the duality is studied in the undeformed gauge theory where $\epsilon_{1}=\epsilon_{2}=0$. Actually, the situation at $-\Lambda^{2}$ is related to that at $\Lambda^{2}$ by a simple map; therefore, we can simplify the following explanation a bit by focusing on the singularity at $\Lambda^{2}$.

In order to determine the prepotential, the dual scalar VEV $a_{D}$ is defined and related to $a$ by the relation $a_{D}=\partial_{a} \mathcal{F}$. Moreover, $a$ and $a_{D}$ are given by integrals of the Seiberg-Witten form along the homology circles $\alpha$ and $\beta$ of the Seiberg-Witten curve determined by the moduli space which is a torus for SU(2) gauge theory, $a=\oint_{\alpha} \lambda_{S W}$ and $a_{D}=\oint_{\beta} \lambda_{S W}$. As $\lambda_{S W}$ depends on the parameters $u, \Lambda^{2}$, in this way we get relations $a\left(u, \Lambda^{2}\right)$ and $a_{D}\left(u, \Lambda^{2}\right)$. The two functions are globally defined on the moduli space; $a\left(u, \Lambda^{2}\right)$ has an asymptotic expansion at $u \sim \infty$ and $a_{D}\left(u, \Lambda^{2}\right)$ has an asymptotic expansion at $u=\Lambda^{2}$.

The gauge theory with $\epsilon_{1} \neq 0, \epsilon_{2}=0$ is the "quantized" version of theory with $\epsilon_{1}=\epsilon_{2}=0$. As first studied in Ref. 31, there is a way to incorporate the $\epsilon_{1}$ deformation into the integrand $\lambda_{S W}$; therefore, we have the quantized Seiberg-Witten form $\lambda_{S W}\left(\epsilon_{1}\right)$. The integrations of $\lambda_{S W}\left(\epsilon_{1}\right)$ along the homology circles of the torus give us the deformed relation $a\left(u, \Lambda^{2}, \epsilon_{1}\right)$ and $a_{D}\left(u, \Lambda^{2}, \epsilon_{1}\right)$. The relation $a\left(u, \Lambda^{2}, \epsilon_{1}\right)$ also has an asymptotic expansion at $u \sim \infty$, by identification (17); the inverse series of the expansion is exactly eigenvalue solution (19). At the point $u=\Lambda^{2}$, the dual relation $a_{D}\left(u, \Lambda^{2}, \epsilon_{1}\right)$ has an asymptotic expansion; it turns out that the inverse series of the expansion gives another eigenvalue solution (23).

The remaining detail is how to compute the deformed integrand $\lambda_{S W}\left(\epsilon_{1}\right)$. As the deformed gauge theory is related to certain quantum mechanics problem, it is not surprising that the WKB method provides an efficient method to obtain higher order quantum corrections. Actually in Ref. 31, it shows that $\lambda_{S W}\left(\epsilon_{1}\right)$ can be generated from the undeformed $\lambda_{S W}$ by the action of certain differential operators with respect to $u$ and $\Lambda^{2}$. In practice, setting $\Lambda^{2}=1$ will further simplify the procedure; it can be restored by dimensional consideration. We use $D\left(u, \partial_{u}, \epsilon_{1}\right)$ to represent the differential operator, then the deformed relation $a\left(u, \epsilon_{1}\right)$ is computed by

$$
a\left(u, \epsilon_{1}\right)=D\left(u, \partial_{u}, \epsilon_{1}\right) \oint_{\alpha} \lambda_{S W} .
$$

The integral $a(u)=\oint_{\alpha} \lambda_{S W}$ is the leading order WKB approximation; higher order $\epsilon_{1}$ corrections are generated by $D\left(u, \partial_{u}, \epsilon_{1}\right)$. The operator $D\left(u, \partial_{u}, \epsilon_{1}\right)$ can be expanded as $D\left(u, \partial_{u}, \epsilon_{1}\right)=$ 
$1+\sum_{n=1}^{\infty} \epsilon_{1}^{n} D_{n}\left(u, \partial_{u}\right)$, and here the $n$ should not be confused with the $n(n-1)$ in the Lamé potential. The differential operators $D_{n}\left(u, \partial_{u}\right)$ are polynomials of $u$ and $\partial_{u}$; the first few of them can be found in Refs. 32 and 33. Restoring the $\Lambda^{2}$ dependence and reversing the large $u$ asymptotic series of $a\left(u, \Lambda^{2}, \epsilon_{1}\right)$, we get the series $u\left(a, \Lambda^{2}, \epsilon_{1}\right)$ which gives eigenvalue (19) through parameters identification (17).

The dual relation $a_{D}\left(u, \epsilon_{1}\right)$ is given by integral of the same deformed integrand along the dual homology circle,

$$
a_{D}\left(u, \epsilon_{1}\right)=D\left(u, \partial_{u}, \epsilon_{1}\right) \oint_{\beta} \lambda_{S W} .
$$

After restoring the dependence on $\Lambda^{2}$, the function $a_{D}\left(u, \Lambda^{2}, \epsilon_{1}\right)$ has an asymptotic series at $u=\Lambda^{2}$ and its reverse series is $u=\left(a_{D}, \Lambda^{2}, \epsilon_{1}\right)$. In order to relate it to the eigenvalue solution of the Mathieu equation, we identify the parameters as

$$
\lambda=\frac{8 u}{\epsilon_{1}^{2}}, \quad v=\frac{2 i a_{D}}{\epsilon_{1}}, \quad h=\frac{4 \Lambda^{2}}{\epsilon_{1}^{2}}=\frac{4 \sqrt{q_{i n}}}{\epsilon_{1}^{2}} .
$$

Then, the asymptotic series expansion of $u\left(a_{D}, \Lambda^{2}, \epsilon_{1}\right)$ gives another well known eigenvalue expansion,

$$
\lambda=2 h-4 v \sqrt{h}+\frac{4 v^{2}-1}{2^{3}}+\frac{4 v^{3}-3 v}{2^{6} \sqrt{h}}+\cdots,
$$

for $h \gg 1, \frac{h}{v^{2}} \gg 1$. The details are given in Ref. 33, see there for result up to the order $h^{-\frac{7}{2}}$. Moreover, the third expansion region near $u=-\Lambda^{2}$, the dyonic region, is mirror of the region near $u=\Lambda^{2}$. The eigenvalue there is obtained from (23) by the map $v \rightarrow i v, h \rightarrow-h$,

$$
\lambda=-2 h+4 v \sqrt{h}-\frac{4 v^{2}+1}{2^{3}}-\frac{4 v^{3}+3 v}{2^{6} \sqrt{h}}+\cdots .
$$

In Appendix $\mathrm{C}$, we give an algorithm that gives the operator $D\left(u, \partial_{u}, \epsilon_{1}\right)$, therefore the deformed $\lambda_{S W}\left(\epsilon_{1}\right)$, up to arbitrary higher order of $\epsilon_{1}$.

\section{COMBINATORIAL APPROACH TO THE LAMÉ EIGENVALUE}

We adopt the same method to the Lamé equation which is quite similar to the Mathieu equation. In the limit $n \rightarrow \infty, k \rightarrow 0$ while keeping $n(n-1) k^{2}=\kappa^{2}$ fixed, the Jacobi $s n$-function becomes the trigonometric function sin, and Eq. (4) becomes equivalent to Eq. (1). According to the argument of quantum field theory, the moduli space of the corresponding $\mathrm{N}=2^{*}$ gauge theory also has three singularities, hence there are three asymptotic expansions for the Lamé eigenvalue. In this section, we derive the eigenvalue expansion at the electric region from the deformed prepotential of gauge theory, and write it in a proper form.

\section{A. Identify parameters}

First, we have to make the identification between the module parameters in the Lamé equation and in the $\mathrm{N}=2^{*}$ gauge theory. The parameter $k$ appearing in Eq. (4) is the elliptic module of the Jacobi elliptic function. The Weierstrass elliptic function uses another module parameter, the nome $q$. The two parameters are related to each other, and their precise relation is

$$
k^{2}=16 q^{\frac{1}{2}}-128 q+704 q^{\frac{3}{2}}-3072 q^{2}+\cdots=\frac{\theta_{2}(q)^{4}}{\theta_{3}(q)^{4}} .
$$

Our claim is that $q$ is exactly the instanton expansion parameter of the $\mathrm{N}=2^{*}$ theory and is the "good" expansion parameter for the eigenvalue $B$ in the electric region, while $k$ is the "good" module parameter for the eigenvalue $A$ in the magnetic and dyonic regions. 
Another thing we have to identify is the relation between the parameter $n$ in the Lamé equation and the adjoint mass of the gauge theory. The equivalent parameter $m$ appears in the instanton partition function of $\mathrm{N}=2^{*}$ theory, as clarified in Ref. 37; the parameter $m$ differs from the physical mass $m^{*}$ by $\epsilon_{1}, \epsilon_{2}$ shift, $m=m^{*}+\left(\epsilon_{1}+\epsilon_{2}\right) / 2$. We identify $n=\epsilon_{1}^{-1} m$.

Last, we have to identify the relation between the eigenvalue $A$, or $B$, and the scalar condensation $u$. The Seiberg-Witten curve of the undeformed $\mathrm{N}=2^{*}$ theory is

$$
y^{2}=\left(x-e_{1} \tilde{u}-\frac{1}{8} e_{1}^{2} m^{2}\right)\left(x-e_{2} \tilde{u}-\frac{1}{8} e_{2}^{2} m^{2}\right)\left(x-e_{3} \tilde{u}-\frac{1}{8} e_{3}^{2} m^{2}\right),
$$

with $\tilde{u}$ related to the VEV of scalar field by

$$
u=\left\langle\operatorname{Tr} \phi^{2}\right\rangle=\tilde{u}-\frac{m^{2}}{24}\left(1-2 E_{2}\right),
$$

where $E_{2}\left(q_{i n}\right)$ is the second Eisenstein series. We have rescaled the mass in Ref. 4 by $m^{2} \rightarrow \frac{m^{2}}{2}$ in order to match the mass parameter that appears in the instanton counting formula. Again this is an elliptic curve, with two conjugate homology circles $\alpha$ and $\beta$, and the Seiberg-Witten differential one form. $\tilde{u}$ and $u$ differ by terms caused by the mass deformation. It turns out that $\tilde{u}$ is directly related to the eigenvalue $B$.

In summary, we identify

$$
B=-\frac{8 \tilde{u}}{\epsilon_{1}^{2}}, \quad v=\frac{2 a}{\epsilon_{1}}, \quad n=\frac{m}{\epsilon_{1}}, \quad q=q_{i n} .
$$

The moduli space has three singularities in the $\tilde{u}$ plane, as showed in Fig. 3; they are located at $\tilde{u}=\frac{1}{8} e_{i} m^{2}, i=1,2,3$. Elliptic curve (26) degenerates at each singularity. Note that the stationary points of the potential $\wp(z)$ are exactly at $z_{1,2,3}$, where $\left.\partial_{z} \wp(z)\right|_{z_{i}}=0,\left.\partial_{z}^{2} \wp(z)\right|_{z_{i}} \neq 0$ and $\wp\left(z_{i}\right)=e_{i}$. In the $u$ plane, the singularities are located at

$$
\begin{aligned}
& u=m^{2}\left(\frac{1}{8}-4 q^{2}-12 q^{4}+12 q^{5}+\cdots\right), \\
& u=\mp m^{2}\left(\sqrt{q} \pm 3 q+4 q^{\frac{3}{2}} \pm 7 q^{2}+\cdots\right) .
\end{aligned}
$$

As in the case for the pure SU(2) SYM (the Mathieu equation), there are only three singularities in the moduli space; therefore, there are only three asymptotic expansion regions for the eigenvalue. While the Mathieu eigenvalue has two free parameters $h$ and $v$, the Lamé eigenvalue has three free parameters, $n, k$, and $v$; it is not a prior fact the number of expansion regions remains to be three. However, the gauge theory argument indicates there is no other expansion region.

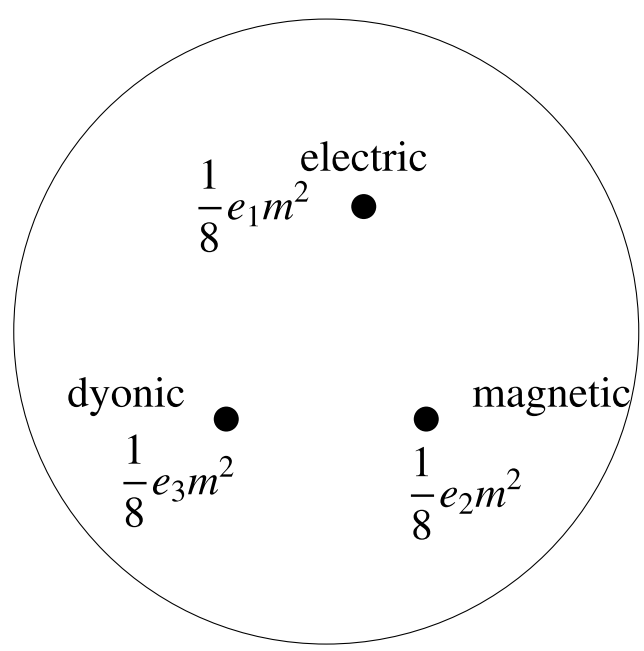

FIG. 3. Singularities in the $\tilde{u}$-plane for $\mathrm{N}=2^{*}$ theory. 


\section{B. Combinatorial evaluating in the electric region}

We derive the eigenvalue in the electric region. Let us look at the perturbative part, in the limit $\epsilon_{2}=0$; the deformed prepotential is given by ${ }^{7}$

$$
\mathcal{F}^{\text {pert }}=\pi i \tau \sum_{i=1}^{2} a_{i}^{2}+2 \pi i \epsilon_{1} \sum_{i, j=1}^{2}\left(\varpi_{\epsilon_{1}}\left(a_{i j}\right)-\varpi_{\epsilon_{1}}\left(a_{i j}-m-\epsilon_{1}\right)\right),
$$

and due to the convention of instanton computation, the variable $a$ for $\mathrm{SU}(2)$ gauge theory is represented by $a_{1}, a_{2}$ satisfying $a_{1}=-a_{2}=a$, while $a_{i j}=a_{i}-a_{j}$ and the complex gauge coupling constant $\tau$ is related to the instanton expansion parameter by $q_{i n}=\exp (2 \pi i \tau)$; the definition of the function $\varpi_{\epsilon_{1}}(x)$ can be found in Ref. 7. Only the first term $a^{2} \ln q_{i n}$ contributes to the eigenvalue.

Then, we come to the instanton part of the gauge theory, or the $q$ correction part of the Lamé eigenvalue. Following the line of instanton counting, we derive the prepotential $\mathcal{F}_{\ell}\left(a_{12}, m, \epsilon_{1}, \epsilon_{2}\right), \ell=$ $1,2,3, \ldots$, and set $\epsilon_{2}=0$; the eigenvalue $B$ can be obtained via the Matone's relation. Without presenting the results of $Z_{\ell}, \ell=1,2,3 \ldots$, which are fairly lengthy, we only give the final results of the prepotential. For example, the 1 -instanton contribution is

$$
\mathcal{F}_{1} q_{\text {in }}=\frac{2 m\left(m-\epsilon_{1}\right)\left[m\left(m-\epsilon_{1}\right)-a_{12}^{2}+\epsilon_{1}^{2}\right]}{a_{12}^{2}-\epsilon_{1}^{2}} q_{\text {in }} .
$$

Writing in terms of $n, v$, and $q$, it is

$$
\mathcal{F}_{1} q_{\text {in }}=\epsilon_{1}^{2} \frac{2 n(n-1) q}{v^{2}-1} \times\left[n(n-1)-\left(v^{2}-1\right)\right] .
$$

In a similar way, we can compute all other coefficients $\mathcal{F}_{\ell}$. A notable fact is that in the final results of instanton counting, the parameter $n$ always appears in the form $n(n-1)$, consistent with its appearance in the Lamé equation. This is already visible in the numerator of counting formula in Ref. 36 where terms involving $m$ combine to $m\left(m-\epsilon_{1}\right)$.

According to the Matone's relation, the scalar condensation $u$ is given by $2 u=q \frac{\partial}{\partial q} \mathcal{F}$, and in the presence of $\epsilon_{1}$ deformation, $\tilde{u}$ and $u$ are related by

$$
\tilde{u}=u+\frac{m\left(m-\epsilon_{1}\right)}{24}\left(1-2 E_{2}(q)\right) \text {. }
$$

Here, we have turned on the $\epsilon_{1}$ quantum correction to the classical relation (27); this is the only consistent way to deform classical relation. Now we are in the position to get the eigenvalue $B$, using identification (28),

$$
\begin{aligned}
B= & -\frac{4}{\epsilon_{1}^{2}} q \frac{\partial}{\partial q} \mathcal{F}-\frac{n(n-1)}{3}\left(1-2 E_{2}(q)\right) \\
= & -v^{2}-\frac{n(n-1)}{3}\left(1-2 E_{2}(q)\right)-\frac{8 n(n-1) q}{v^{2}-1}\left[n(n-1)-\left(v^{2}-1\right)\right] \\
& -\frac{8 n(n-1) q^{2}}{\left(v^{2}-1\right)^{3}\left(v^{2}-4\right)} \times\left[n^{3}(n-1)^{3}\left(5 v^{2}+7\right)-12 n^{2}(n-1)^{2}\left(v^{2}-1\right)^{2}\right. \\
& \left.+6 n(n-1)\left(v^{2}-1\right)^{2}\left(v^{2}-2\right)-3\left(v^{2}-1\right)^{3}\left(v^{2}-4\right)\right]+O\left(q^{3}\right) .
\end{aligned}
$$

The term $-v^{2}$ comes from the perturbative result $q \partial_{q} \mathcal{F}^{\text {pert }}=\epsilon_{1}^{2} v^{2} / 4$. Expansion (34) for $B$ is valid when

$$
q \ll 1, \quad n \gg 1, \quad v \gg 1, \quad n q^{\frac{1}{4}} \ll v .
$$

Close to the electric singularity, we have the leading order behavior $8 u \sim m^{2}$, that is $v^{2} \sim n(n-1)$, therefore $B \sim-\frac{2}{3} n(n-1)+O(n(n-1) q)$. An equivalent expansion was also obtained by Langmann in Ref. 14 by a different method. We have shown the equivalence of his eigenvalue expansion $\mathcal{E}$ and our expansion $B$, see Appendix B.

At this point, we should emphasise a subtle point, as explained in Ref. 42; the Nekrasov partition function actually computes the instanton effects of $\mathrm{U}(2)$ gauge theory; even after setting 
$a_{1}=-a_{2}=a$ due to the traceless condition of $\mathrm{SU}(2)$, it differs from $\mathrm{SU}(2)$ theory by an $\mathrm{U}(1)$ factor. Therefore, the deformed prepotential $\mathcal{F}$ in this subsection should be understood as $U(2)$ prepotential subject to the traceless condition; it differs from the SU(2) prepotential by the value $\frac{1}{12} m\left(m-\epsilon_{1}\right)\left(1-E_{2}\right)$. There is no such $\mathrm{U}(1)$ factor difference for pure gauge theories with gauge groups $\mathrm{U}(2)$ and $\mathrm{SU}(2)$.

In order to compare (34) with the WKB results, we rewrite the asymptotic expansion in terms of $A(\mu, k)$. It can be derived from the expansion of $B$, taking into account the relation between $A$ and $B$ in (8), the relation between $\mu$ and $v$ in (12), and the relation between $k$ and $q$ in (25). We get

$$
\begin{aligned}
A= & -\mu^{2}-\frac{1}{2} n(n-1) k^{2}-n(n-1)\left(\frac{1}{16} k^{4}+\frac{1}{32} k^{6}+\frac{41}{2048} k^{8}+\frac{59}{4096} k^{10}+\cdots\right) \\
& -\frac{n^{2}(n-1)^{2}}{\mu^{2}}\left[\frac{1}{32} k^{4}-\frac{1}{4096} k^{8}-\frac{1}{4096} k^{10}+\cdots\right] \\
& -\frac{n^{2}(n-1)^{2}}{\mu^{4}}\left[\frac{1}{32} k^{4}-\frac{1}{64} k^{6}-\left(\frac{7+6 n(n-1)}{4096}\right) k^{8}-\left(\frac{7+6 n(n-1)}{8192}\right) k^{10}+\cdots\right] \\
& +O\left(\frac{1}{\mu^{6}}\right) .
\end{aligned}
$$

Before doing the WKB check, we first give a simple consistent check using a limit of the Lamé equation. The $\mathrm{N}=2^{*}$ theory has a decoupling limit. When the energy scale is very small, or the mass is very large, the adjoint mass decouples and the theory flows to the pure gauge theory. The limit is $q \rightarrow 0, m \rightarrow \infty$ with $m^{2} \sqrt{q} \rightarrow-\Lambda^{2}$ or equivalently $k \rightarrow 0, m \rightarrow \infty$, with $m^{2} k^{2} \rightarrow-16 \Lambda^{2}$. In this limit, every singularity in the moduli space of the $\mathrm{N}=2^{*}$ theory becomes a singularity of the pure gauge theory. This can be clearly seen in (29). Writing in terms of the parameters of the differential equation, the limit is $q \rightarrow 0, n \rightarrow \infty$ with $n(n-1) \sqrt{q} \rightarrow-\frac{h}{4}$ or equivalently $k \rightarrow 0, n \rightarrow \infty$ with $n(n-1) k^{2} \rightarrow-4 h$. Accordingly, in this limit, the Lamé equation reduces to the Mathieu equation, and the Lamé eigenvalue expansions (34) and (36) reduce to Mathieu eigenvalue expansion (19), $-B \rightarrow \lambda-\frac{1}{3} n(n-1),-A \rightarrow \lambda-2 h$.

\section{EIGENVALUE FROM THE WKB ANALYSIS}

We perform the WKB perturbation to confirm that the asymptotic expansions in (34) and (36) are indeed eigenvalue solutions for the Lamé equation. The method is explained in Section III, for some periodic potentials including the elliptic potential; the higher order WKB perturbations are generated from the leading order by the action of differential operators. ${ }^{31}$ In this section, we do the same thing for the Lamé equation, as have done for the Mathieu equation in Refs. 31-33.

Equation (4) is rewritten as

$$
\frac{\epsilon^{2}}{2} \Phi^{\prime \prime}-\left(\omega+\operatorname{sn}^{2} \varkappa\right) \Phi=0
$$

where

$$
\epsilon^{2}=\frac{2}{n(n-1) k^{2}}, \quad \omega=\frac{A}{n(n-1) k^{2}} .
$$

Note that $\epsilon_{1}$ is contained in $\epsilon$. Suppose $\epsilon \ll 1$, i.e., $n q^{\frac{1}{4}} \gg 1$, then $\epsilon$ is a small parameter for the WKB expansion. We choose Eq. (4) to do the WKB analysis because there are readily available integral formulae for the Jacobi elliptic functions. It is legal to choose Eq. (5) instead, as in Refs. 28 and 38 where the leading order expansion is performed. Expanding the function $\Phi$ as WKB series,

$$
\Phi(\varkappa)=\exp i \int_{\varkappa_{0}}^{\varkappa} d \varkappa^{\prime}\left(\frac{p_{0}\left(\varkappa^{\prime}\right)}{\epsilon}+p_{1}\left(\varkappa^{\prime}\right)+\epsilon p_{2}\left(\varkappa^{\prime}\right)+\epsilon^{2} p_{3}\left(\varkappa^{\prime}\right)+\cdots\right),
$$


then substituting into the equation, $p_{n}$ can be determined recursively,

$$
\begin{aligned}
& p_{0}=i \sqrt{2} \sqrt{\omega+\operatorname{sn}^{2} \varkappa}, \quad p_{1}=\frac{i}{2}\left(\ln \left(p_{0}\right)\right)^{\prime}, \quad p_{2}=\frac{1}{4 p_{0}}\left[\frac{3}{2}\left(\frac{p_{0}^{\prime}}{p_{0}}\right)^{2}-\frac{p_{0}^{\prime \prime}}{p_{0}}\right], \\
& p_{3}=\frac{i}{2}\left(\frac{p_{2}}{p_{0}}\right)^{\prime}, \quad p_{4}=\frac{i}{2}\left(\frac{p_{3}}{p_{0}}\right)^{\prime}-\frac{p_{2}^{2}}{2 p_{0}}, \quad \cdots,
\end{aligned}
$$

where the prime denotes $\frac{\partial}{\partial x}$.

According to the Floquet theory, in order to obtain the dispersion relation $A(\mu)$, we have to evaluate the contour integral $\oint_{\alpha} p d x$, where $\alpha$ is the homology cycle of curve (26) relevant to electric scalar VEV. Let us first carry out the integral for the leading order integrand $\oint_{\alpha} p_{0} d x$. The integral can be computed using the amplitude, defined by $\varphi=\operatorname{am} \varkappa$, then we have

$$
\operatorname{sn} \varkappa=\sin \varphi, \quad d \varkappa=\frac{d \varphi}{\sqrt{1-k^{2} \sin ^{2} \varphi}} .
$$

The integral becomes

$$
\oint_{\alpha} \sqrt{\omega+\operatorname{sn}^{2} \varkappa} d x=2 \int_{0}^{K} \sqrt{\omega+\operatorname{sn}^{2} \varkappa} d x=2 \int_{0}^{\frac{\pi}{2}} \sqrt{\frac{\omega+\sin ^{2} \varphi}{1-k^{2} \sin ^{2} \varphi}} d \varphi .
$$

In the electric region, $A \gg \kappa^{2}$, therefore $\omega \gg 1$, we have the expansion

$$
\sqrt{\omega+\sin ^{2} \varphi}=\sqrt{\omega}\left(1+\frac{\sin ^{2} \varphi}{2 \omega}-\frac{\sin ^{4} \varphi}{8 \omega^{2}}+\frac{\sin ^{6} \varphi}{16 \omega^{3}}+\cdots\right) .
$$

All the integrals we need to do are of the form

$$
\int_{0}^{\frac{\pi}{2}} \frac{\sin ^{2 n} \varphi}{\Delta} d \varphi, \quad \text { with } \quad \Delta=\sqrt{1-k^{2} \sin ^{2} \varphi}, \quad n=0,1,2,3, \ldots
$$

They are given in Chapter 2 of Ref. 39. After collecting all terms together, we get

$$
\begin{aligned}
\oint_{\alpha} \sqrt{\omega+\mathrm{sn}^{2} \varkappa} d \varkappa= & 2 K \sqrt{\omega}+\frac{K-E}{k^{2}} \omega^{-1 / 2}-\frac{\left(2+k^{2}\right) K-2\left(1+k^{2}\right) E}{12 k^{4}} \omega^{-3 / 2} \\
& +\frac{\left(8+3 k^{2}+4 k^{4}\right) K-\left(8+7 k^{2}+8 k^{4}\right) E}{120 k^{6}} \omega^{-5 / 2}+\cdots,
\end{aligned}
$$

where $K$ and $E$ are the complete elliptic integrals of the first and second kind, respectively.

For higher order contour integrals, similar to the case of Mathieu equation, they can be obtained from higher order differential operators acting on $\oint_{\alpha} p_{0} d x$,

$$
\oint_{\alpha} p_{n} d x=D_{n}\left(\omega, \partial_{\omega}, k\right) \oint_{\alpha} p_{0} d x
$$

The odd order integrals actually vanish because the integrand are total derivatives,

$$
\oint_{\alpha} p_{2 l+1} d x=0, \quad l=0,1,2 \ldots
$$

Hence, we can set all odd differential operators $D_{2 l+1}\left(\omega, \partial_{\omega}, k\right)=0$. While for the even order integrals, using the trick of Ref. 31, we get the operator $D_{2 l}\left(\omega, \partial_{\omega}, k\right)$. For example, we get $D_{2}\left(\omega, \partial_{\omega}, k\right)$ from

$$
\begin{aligned}
\oint_{\alpha} p_{2} d x= & -\frac{1}{12}\left[\left(1+2 \omega+2 \omega k^{2}+3 \omega^{2} k^{2}\right) \partial_{\omega}^{2}\right. \\
& \left.+\left(1+k^{2}+3 \omega k^{2}\right) \partial_{\omega}-\frac{3}{4} k^{2}\right] \oint_{\alpha} p_{0} d x
\end{aligned}
$$


And $D_{4}\left(\omega, \partial_{\omega}, k\right)$ is given by

$$
\begin{aligned}
\oint_{\alpha} p_{4} d \varkappa= & \frac{1}{64}\left\{\frac { 2 } { 1 3 5 } \left[21+\left(84+359 k^{2}\right) \omega+\left(84+1394 k^{2}+359 k^{4}\right) \omega^{2}\right.\right. \\
& \left.+k^{2}\left(1077+1352 k^{2}\right) \omega^{3}+1014 k^{4} \omega^{4}\right] \partial_{\omega}^{4} \\
& +\frac{4}{27}\left[\left(18+73 k^{2}\right)+\left(36+341 k^{2}+146 k^{4}\right) \omega+k^{2}\left(432+597 k^{2}\right) \omega^{2}+597 k^{4} \omega^{3}\right] \partial_{\omega}^{3} \\
& +\frac{1}{18}\left[\left(60+191 k^{2}+225 k^{4}\right)+k^{2}\left(667 k+1162 k^{2}\right) \omega+1743 k^{4} \omega^{2}\right] \partial_{\omega}^{2} \\
& \left.+\frac{1}{18}\left[k^{2}\left(8+63 k^{2}\right)+189 k^{4} \omega\right] \partial_{\omega}-\frac{k^{4}}{8}\right\} \oint_{\alpha} p_{0} d x .
\end{aligned}
$$

In Appendix C, we give a systematic method to obtain higher order differential operators.

The contour integral along the $\alpha$ circle computes the phase shift of the function $\Phi(\varkappa)$ in (10); we have

$$
2 K \mu=\oint_{\alpha} d \varkappa p(\varkappa)=\left(1+\sum_{n=1}^{\infty} \epsilon^{n} D_{n}\left(\omega, \partial_{\omega}, k\right)\right) \oint_{\alpha} d x \frac{p_{0}(\varkappa)}{\epsilon} .
$$

Therefore, the Floquet exponent is given by

$$
\mu=\frac{1}{2 K} \oint_{\alpha} d \varkappa p(\varkappa)
$$

The remaining work is straightforward but tedious: expand the $p_{0}$ integral (45) as power series of $\omega$ and $k$, use the differential operators $D_{2 l}\left(\omega, \partial_{\omega}, k\right)$ to generate higher order integrals for $p_{2}, p_{4}, \ldots$, hence we get the series expansion of $\mu=\mu(\omega, k)$. Then, inverse the series $\mu(\omega, k)$ to get a series $\omega=\omega(\mu, k)$; we finally get the series expansion for the eigenvalue $A$ using $A=n(n-1) k^{2} \omega$. We have checked it indeed gives expansion (36).

\section{EXTENSION TO OTHER ASYMPTOTIC EXPANSION REGIONS}

\section{A. Magnetic expansion}

The last task we have to do is extending the asymptotic expansion in the electric region to the magnetic and dyonic regions, as what we have done for the Mathieu equation in Ref. 33. We stress that the asymptotic expansion in the magnetic region has been worked out in the literature, using purely mathematical technique. The explicit formula is given in the book by Müller-Kirsten, ${ }^{12}$ which cites results from earlier original paper of Ince and paper of Müller. It is also presented in Chapter 29 of Ref. 11. It expands as

$$
\begin{aligned}
A= & -i 2 \kappa \mu-\frac{1}{2^{3}}\left(1+k^{2}\right)\left(4 \mu^{2}-1\right)-\frac{i}{2^{5} \kappa}\left[\left(1+k^{2}\right)^{2}\left(4 \mu^{3}-3 \mu\right)-4 k^{2}\left(4 \mu^{3}-5 \mu\right)\right] \\
& +\frac{1}{2^{10} \kappa^{2}}\left(1+k^{2}\right)\left(1-k^{2}\right)^{2}\left(80 \mu^{4}-136 \mu^{2}+9\right)+\cdots .
\end{aligned}
$$

The expansion is valid for $n k \gg 1, n k \gg \mu$. Note $k \sim q^{\frac{1}{4}}$. We remind the readers about the notation difference; the Floquet exponent in Ref. 12 is denoted by $q$, and its relation to our $\mu$ is $q=2 i \mu$. Also there the eigenvalue is denoted by $\Lambda$, related to ours by $\Lambda=-A$. In the limit $n \rightarrow \infty, k \rightarrow 0$ while keeping $\kappa^{2}=n(n-1) k^{2} \rightarrow-4 h$ fixed, eigenvalue (52) reduces to corresponding Mathieu eigenvalue (23), $-A \rightarrow \lambda-2 h$.

Now, let us look at how to extend Lamé eigenvalue (36) in the electric region to the magnetic region. The tool is the differential operators obtained in Sec. $\mathrm{V}$, and apply them to the $\beta$-contour integral of $p_{0}$. In the magnetic region located near $A \sim 0$, where $\omega \ll 1$, we can expand $\sqrt{\omega+\sin ^{2} \varphi}$ as

$$
\sqrt{\omega+\sin ^{2} \varphi}=\sin \varphi+\frac{\omega}{2} \frac{1}{\sin \varphi}-\frac{\omega^{2}}{8} \frac{1}{\sin ^{3} \varphi}+\frac{\omega^{3}}{16} \frac{1}{\sin ^{5} \varphi}+\cdots
$$


All the integrals now we need to do are

$$
\oint_{\beta} \frac{1}{\Delta \sin ^{2 n-1} \varphi} d \varphi=2 \int_{\frac{\pi}{2}}^{\varphi_{0}} \frac{1}{\Delta \sin ^{2 n-1} \varphi} d \varphi, \quad n=0,1,2,3, \ldots,
$$

with $\sin \varphi_{0}=\frac{1}{k}$; note that both $\varphi_{0}$ and $k$ are complex quantities. They are also given in Chapter 2 of Ref. 39. The first few orders of the integral $\oint_{\beta} p_{0} d \varkappa$ give

$$
\oint_{\beta} \sqrt{\omega+\operatorname{sn}^{2} \varkappa} d \varkappa=i \pi\left(\frac{1}{2} \omega-\frac{1+k^{2}}{16} \omega^{2}+\frac{3 k^{4}+2 k^{2}+3}{128} \omega^{3}+\cdots\right) .
$$

Using the differential operators obtained in Sec. V, we can generate integrals $\oint_{\beta} p_{n} d \varkappa$ for $n \geqslant 2$.

For the Floquet exponent in the magnetic region, our claim is

$$
\mu=\frac{1}{i \pi} \oint_{\beta} d x p(x)
$$

There is a point a bit puzzling. When we go through the circle $\beta$, the coordinate $\varkappa$ shifts $2 i K^{\prime}=$ $2 i K\left(k^{\prime}\right)$; according to the Floquet theory, the exponent is defined by the relation $2 i K\left(k^{\prime}\right) \mu=\oint_{\beta} p d x$. Therefore, the phase $\oint_{\beta} p d \varkappa$ should be divided by $2 i K^{\prime}$ to get the exponent $\mu$. But we find the correct number to divide by is $i \pi$, in order to reproduce known expansion (52).

The remaining work is the same as that in Sec. V: use the differential operators $D_{2 l}\left(\omega, \partial_{\omega}, k\right)$ to generate higher order integrals for $p_{2}, p_{4}, \ldots$, so we get the series expansion of $\mu=\mu(\omega, k)$. Then, inverse the series $\mu(\omega, k)$ to get a series $\omega=\omega(\mu, k)$. Finally, we get the series expansion for the eigenvalue $A$ using $A=n(n-1) k^{2} \omega=\kappa^{2} \omega$. With the algorithm in Appendix C, we have reproduced expansion (52) up to higher order.

From the magnetic expansion $A(\mu, k)$ given above, use relation (8) and change parameters $\mu, k$ to $v, q$; we get the magnetic expansion of $B(v, q)$. Of course, $B(v, q)$ is not economic for the magnetic expansion.

\section{B. Dyonic expansion}

The eigenvalue at the dyonic point is mirror to the one at the magnetic point. This is most obvious for $B(v, q) \sim \tilde{u}$. From Fig. 3, we know that the magnetic point $\tilde{u}=e_{2} m^{2} / 8$ is mapped to the dyonic point $\tilde{u}=e_{3} m^{2} / 8$ by $e_{2} \rightarrow e_{3}$, or $q^{\frac{1}{2}} \rightarrow-q^{\frac{1}{2}}$. Also from the story of Mathieu equation, ${ }^{32}$ we know that the exponent of magnetic region is mapped to the exponent of dyonic region by $v \rightarrow i v$. Therefore by the mirror map $q^{\frac{1}{2}} \rightarrow-q^{\frac{1}{2}}, v \rightarrow i v$, we get the dyonic expansion $B_{d}$ from its magnetic expansion $B_{m}$.

It is also interesting to look at how the dyonic expansion of $A$ can be obtained from its magnetic expansion. For this purpose, we need to know how the other parameters are changed by the map. It is simple to see that under $q^{\frac{1}{2}} \rightarrow-q^{\frac{1}{2}}$, we have $e_{2}$ and $e_{3}$ interchanged while $e_{1}$ unchanged. Furthermore, since

$$
k=4 q^{\frac{1}{4}} \prod_{n=1}^{\infty}\left(\frac{1+q^{n}}{1+q^{n-\frac{1}{2}}}\right)^{4}, \quad k^{\prime}=\prod_{n=1}^{\infty}\left(\frac{1-q^{n-\frac{1}{2}}}{1+q^{n-\frac{1}{2}}}\right)^{4},
$$

the map leads to a simple change for $k$ and $k^{\prime}$,

$$
k \rightarrow i \frac{k}{k^{\prime}}, \quad k^{\prime} \rightarrow \frac{1}{k^{\prime}}
$$

For the magnetic expansion, $A$ and $B$ satisfy

$$
\begin{aligned}
B_{m} & =\left(e_{1}-e_{2}\right) A_{m}(\mu, k)-e_{2} n(n-1) \\
& =\left(e_{1}-e_{2}\right) A_{m}\left(\frac{v}{\sqrt{e_{1}-e_{2}}}, k(q)\right)-e_{2} n(n-1) .
\end{aligned}
$$


The mirror map changes the relation to

$$
B_{d}=\left(e_{1}-e_{3}\right) A_{m}\left(\frac{i v}{\sqrt{e_{1}-e_{3}}}, i \frac{k}{k^{\prime}}\right)-e_{3} n(n-1) .
$$

The subscripts $m, d$ are used to emphasize that $A, B$ should be understood as asymptotic series in magnetic and dyonic regions. Then substituting this into the dyonic relation $B_{d}=\left(e_{1}-\right.$ $\left.e_{2}\right) A_{d}(\mu, k)-e_{2} n(n-1)$, we get

$$
\begin{aligned}
A_{d}(\mu, k) & =\frac{e_{1}-e_{3}}{e_{1}-e_{2}} A_{m}\left(\frac{i v}{\sqrt{e_{1}-e_{3}}}, i \frac{k}{k^{\prime}}\right)-\frac{e_{3}-e_{2}}{e_{1}-e_{2}} n(n-1) \\
& =\frac{e_{1}-e_{3}}{e_{1}-e_{2}} A\left(i \mu \sqrt{\frac{e_{1}-e_{2}}{e_{1}-e_{3}}}, i \frac{k}{k^{\prime}}\right)-\frac{e_{3}-e_{2}}{e_{1}-e_{2}} n(n-1),
\end{aligned}
$$

where the function $A$ is magnetic expansion (52) given in Subsection VI A. Taking into account the relation of $k^{2}$ and $e_{i}$ in (A5), we obtain

$$
A_{d}=k^{\prime 2} A\left(\frac{i \mu}{k^{\prime}}, \frac{i k}{k^{\prime}}\right)-k^{2} n(n-1) .
$$

The first few terms expand as

$$
\begin{aligned}
A_{d}= & -\kappa^{2}+i 2 \kappa \mu+\frac{1}{2^{3}}\left(1-2 k^{2}\right)\left(\frac{4 \mu^{2}}{k^{\prime 2}}+1\right) \\
& +\frac{i}{2^{5} \kappa}\left[\frac{\left(1-2 k^{2}\right)^{2}}{k^{\prime}}\left(\frac{4 \mu^{3}}{k^{\prime 3}}+\frac{3 \mu}{k^{\prime}}\right)+4 k^{2} k^{\prime}\left(\frac{4 \mu^{3}}{k^{\prime 3}}+\frac{5 \mu}{k^{\prime}}\right)\right]+\cdots
\end{aligned}
$$

In the decoupling limit, $A_{d}$ reduces to the corresponding Mathieu eigenvalue (24), $-A_{d} \rightarrow \lambda-2 h$.

We can perform the WKB analysis to confirm the above argument. Set $A=-\kappa^{2}+\widetilde{A}$, i.e., $\omega=$ $-1+\widetilde{\omega}$, then Lamé equation (37) becomes

$$
\frac{\epsilon^{2}}{2} \Phi^{\prime \prime}-\left(\widetilde{\omega}-\mathrm{cn}^{2} \varkappa\right) \Phi=0
$$

The WKB analysis for this equation is very similar as that in the magnetic case, now with the leading order WKB integrand $\widetilde{p}_{0}=i \sqrt{2} \sqrt{\widetilde{\omega}-\mathrm{cn}^{2} \varkappa}$, and the integral contour is $\gamma=\alpha+\beta$. Using the amplitude variable, the integration is

$$
(i \sqrt{2})^{-1} \oint_{\gamma} \widetilde{p}_{0} d x=2 \int_{0}^{K+i K^{\prime}} \sqrt{\widetilde{\omega}-\operatorname{cn}^{2} \varkappa} d x=2 \int_{0}^{\varphi_{0}} \sqrt{\frac{\widetilde{\omega}-\cos ^{2} \varphi}{1-k^{2} \sin ^{2} \varphi}} d \varphi .
$$

In the dyonic region, the eigenvalue is expanded for $\kappa \gg 1, \kappa \gg \mu$, i.e., $\widetilde{\omega} \ll 1$; therefore, using

$$
\sqrt{\widetilde{\omega}-\cos ^{2} \varphi}=i\left(\cos \varphi-\frac{\widetilde{\omega}}{2} \frac{1}{\cos \varphi}-\frac{\widetilde{\omega}^{2}}{8} \frac{1}{\cos ^{3} \varphi}-\frac{\widetilde{\omega}^{3}}{16} \frac{1}{\cos ^{5} \varphi}+\cdots\right),
$$

the integrals we need to do are

$$
\oint_{\gamma} \frac{1}{\Delta \cos ^{2 n-1} \varphi} d \varphi=2 \int_{0}^{\varphi_{0}} \frac{1}{\Delta \cos ^{2 n-1} \varphi} d \varphi, \quad n=0,1,2,3, \ldots
$$

The first few of them give

$$
\oint_{\gamma} \sqrt{\widetilde{\omega}-\mathrm{cn}^{2} \varkappa} d \varkappa=-\pi\left(\frac{1}{2 k^{\prime}} \widetilde{\omega}+\frac{1-2 k^{2}}{16 k^{\prime 3}} \widetilde{\omega}^{2}+\frac{8 k^{4}-8 k^{2}+3}{128 k^{\prime 5}} \widetilde{\omega}^{3}+\cdots\right) .
$$

For the higher order WKB integrals, they are given by the differential operators $\widetilde{D}_{n}\left(\widetilde{\omega}, \partial_{\widetilde{\omega}}, k\right)$ acting on the leading order integral. As in Subsection VI A, for odd order integrals, $\oint_{\gamma} \widetilde{p}_{2 l+1} d x=0$; therefore, we set $\widetilde{D}_{2 l+1}\left(\widetilde{\omega}, \partial_{\widetilde{\omega}}, k\right)=0$. For even order integrals, we get nontrivial $\widetilde{D}_{2 l}\left(\widetilde{\omega}, \partial_{\widetilde{\omega}}, k\right)$, for 
example, $\widetilde{D}_{2}\left(\widetilde{\omega}, \partial_{\widetilde{\omega}}, k\right)$ is obtained from

$$
\begin{aligned}
\oint_{\gamma} \widetilde{p}_{2} d x= & -\frac{1}{12}\left[\left(-1+k^{2}+2 \widetilde{\omega}-4 k^{2} \widetilde{\omega}+3 k^{2} \widetilde{\omega}^{2}\right) \partial_{\widetilde{\omega}}^{2}\right. \\
& \left.+\left(1-2 k^{2}+3 k^{2} \widetilde{\omega}\right) \partial_{\widetilde{\omega}}-\frac{3}{4} k^{2}\right] \oint_{\gamma} \widetilde{p}_{0} d x .
\end{aligned}
$$

Appendix $\mathrm{C}$ gives the algorithm to obtain all $\widetilde{D}_{2 l}\left(\widetilde{\omega}, \partial_{\widetilde{\omega}}, k\right)$, in a simpler way they can also be obtained from $D_{2 l}\left(\omega, \partial_{\omega}, k\right)$ using property (72) explained below.

We find that for the dyonic expansion, the Floquet exponent is given by

$$
\mu=\frac{k^{\prime}}{\pi} \oint_{\gamma} d x \widetilde{p}(\varkappa)
$$

The factor $k^{\prime} / \pi$ also does not follow the conventional definition of the Floquet exponent; at the moment we do not have a satisfying explanation for this, but the relation is indeed satisfied. We check this relation against (62) up to high order.

Some interesting phenomena are observed and deserve mentioned. Look at the leading order contour integrals for the magnetic and dyonic expansions, formulae (55) and (68); they are related in a simple way,

$$
k \rightarrow i \frac{k}{k^{\prime}}, \quad \omega \rightarrow-\widetilde{\omega} \quad \text { leads to } \quad \frac{1}{\epsilon} \oint_{\beta} \sqrt{\omega+\operatorname{sn}^{2} \varkappa} d x \rightarrow \frac{-1}{\epsilon} \oint_{\gamma} \sqrt{\widetilde{\omega}-\operatorname{cn}^{2} \varkappa} d x .
$$

Note that $\epsilon \sim K^{-1} \sim k^{-1}$ also changes as $\epsilon \rightarrow-i k^{\prime} \epsilon$. Then, look at the differential operators, formulae (48) and (69); they are also related in a simple way,

$$
k \rightarrow i \frac{k}{k^{\prime}}, \quad \omega \rightarrow-\widetilde{\omega} \quad \text { leads } \quad \text { to } \quad \epsilon^{n} D_{n}\left(\omega, \partial_{\omega}, k\right) \rightarrow \epsilon^{n} \widetilde{D}_{n}\left(\widetilde{\omega}, \partial_{\widetilde{\omega}}, k\right), \quad n=2 .
$$

The relation continues to hold for larger $n$. Combining the two facts, under the mirror map, we have

$$
\oint_{\beta} d x p(x) \rightarrow-\oint_{\gamma} d x \widetilde{p}(x), \quad \text { i.e., } \quad \mu \rightarrow i \frac{\mu}{k^{\prime}} .
$$

This is consistent with (62) where the Floquet exponent appears as $i \mu / k^{\prime}$.

\section{CONCLUSION}

Combinatorics is a very interesting and fruitful subject of mathematics; its relation to integrable models is not new. ${ }^{40}$ In recent years, some progresses continue to reveal its fascinating connection to quantum field theory, integrable model, and string/M theory. ${ }^{7,23,41,42}$ Based on a relation between $\mathrm{N}=2$ gauge theory and quantum integrable system, ${ }^{7}$ we provide a quantum field theory approach to spectral problem of the Mathieu equation and the Lamé equation. The approach is combinatorial, based on the instanton counting method. ${ }^{22}$ We use the electro-magnetic duality in gauge theory to derive all three asymptotic expansions of eigenvalue.

Our main results are about the gauge theory explanation of asymptotic expansion formulae (34), (52), and (62) for the eigenvalue of the Lamé equation; the discussion relies on the Floquet theory of periodic differential equation. While expansions (34) and (52) were obtained by some other independent methods, to our knowledge, expansion (62) is a new result. Langmann's paper Ref. 14 gives an expansion equivalent to (34); we have shown the equivalence in Appendix B. We perform the WKB analysis in Sections V and VI, given all three expansions. Also in the decoupling limit, the expansions correctly reduce to expansions of the Mathieu eigenvalue.

In this paper, we do not discuss the combinatorial connection to the eigenfunctions: the Mathieu function and Lamé function/polynomial. There are examples for some integrable models; a full combinatorial approach to the eigenvalue and eigenstate exists. The Calogero-Sutherland model is a famous example, see a recent discussion in Ref. 43, and relevant references therein. In fact, the Calogero-Sutherland model is the trigonometric limit of the elliptic Calogero-Moser model. 
It is argued that in the context of Alday-Gaiotto-Tachikawa(AGT) relation, ${ }^{42}$ the instanton partition function of gauge theory with full surface operator, in the semiclassical limit $\epsilon_{2} \rightarrow 0$, gives the eigenfunction of the corresponding quantum integrable model..$^{27,28}$ This maybe helpful for an explicit combinatorial construction of the eigenfunction.

While the basic tools of the paper, such as the Seiberg-Witten theory and instanton calculation, have been extensively studied by mathematical physicists and have a solid mathematical foundation, some points of our results lack rigorous mathematical proof. This includes the precise meaning of the potential $\mathcal{F}$ for the differential equations, the relation between the instanton method and Langmann's method. Another unsatisfying point is about the puzzling factors $(i \pi)^{-1}$ and $k^{\prime}(\pi)^{-1}$ in relations (56) and (70). In our paper, Ref. 47 we use a different method to study the asymptotic expansions for the eigenvalue of Schrödinger operator with elliptic potentials, including a new example of the ellipsoidal wave equation, find the same factors are needed to define the Floquet exponent in order to produce asymptotic expansions consistent with known results. These results suggest that the Floquet theory for doubly periodic elliptic potentials requires a new treatment unrecorded in conventional Floquet theory. Therefore, we do not present a mathematically rigorous treatment of the Mathieu and the Lamé equations in this single paper, but just to show how the quantum field theories are related to these classical differential equations and can be used to obtain some consistent results of them. We hope to clarify some of the points in the future.

\section{ACKNOWLEDGMENTS}

During the progress of this work I was supported by NSFC No. 11031005 through ZJU, China; later, I was supported by FAPESP No. 2011/21812-8 through IFT-UNESP, Brazil. I thank an anonymous referee for bringing Langmann's results, Ref. 14, to my attention and suggestions for better presentation of the paper.

\section{APPENDIX A: MODULAR FUNCTIONS AND THETA CONSTANTS}

We collect some basic facts about modular functions we have used in this paper. Our main references are Refs. 44-46.

The complete elliptic integrals of the first kind is defined by

$$
K=\int_{0}^{\frac{\pi}{2}} \frac{d \varphi}{\sqrt{1-k^{2} \sin ^{2} \varphi}}=\frac{\pi}{2} F\left(\frac{1}{2}, \frac{1}{2}, 1 ; k^{2}\right),
$$

where $F(a, b, c ; x)$ is the hypergeometric function. The complete elliptic integrals of the second kind is defined by

$$
E=\int_{0}^{\frac{\pi}{2}} d \varphi \sqrt{1-k^{2} \sin ^{2} \varphi}=\frac{\pi}{2} F\left(-\frac{1}{2}, \frac{1}{2}, 1 ; k^{2}\right)
$$

Then, we define the elliptic nome $q=e^{2 \pi i \tau}$ through $\tau=\frac{i K^{\prime}}{K}$, where $K^{\prime}=K\left(k^{\prime}\right)$. The theta constant $\theta_{i}$, which are the theta function $\theta_{i}(z ; q)$ at $z=0$, are given by

$$
\begin{aligned}
& \theta_{1}(q)=0, \quad \theta_{2}(q)=2 q^{\frac{1}{8}} \sum_{n=0}^{\infty} q^{\frac{n(n+1)}{2}}, \\
& \theta_{3}(q)=1+2 \sum_{n=1}^{\infty} q^{\frac{n^{2}}{2}}, \quad \theta_{4}(q)=1+2 \sum_{n=1}^{\infty}(-1)^{n} q^{\frac{n^{2}}{2}} .
\end{aligned}
$$

They satisfy the relation $\theta_{3}^{4}=\theta_{2}^{4}+\theta_{4}^{4}$.

From the theta constant, we define

$$
e_{1}=\frac{2}{3} \theta_{3}^{4}-\frac{1}{3} \theta_{2}^{4}, \quad e_{2}=-\frac{1}{3}\left(\theta_{3}^{4}+\theta_{2}^{4}\right), \quad e_{3}=-\frac{1}{3} \theta_{3}^{4}+\frac{2}{3} \theta_{2}^{4} .
$$


$e_{i}$ satisfy $e_{1}+e_{2}+e_{3}=0$; they are related to the Weierstrass function, and the roots $z_{i}$ of $\wp^{\prime 2}(z)=$ $4 \wp^{3}(z)-g_{2} \wp(z)-g_{3}=0$ satisfy $\wp\left(z_{i}\right)=e_{i}$. We have the following relation between $k$ and $q$ :

$$
k^{2}=\frac{e_{3}-e_{2}}{e_{1}-e_{2}}=\frac{\theta_{2}^{4}}{\theta_{3}^{4}},
$$

which is equivalent to the relation in (25).

The second Eisenstein series is represented by

$$
\begin{aligned}
E_{2}(q) & =1-24 \sum_{n=1}^{\infty} \frac{n q^{n}}{1-q^{n}}=1-24 \sum_{n=1}^{\infty} \frac{q^{n}}{\left(1-q^{n}\right)^{2}} \\
& =1-24 q-72 q^{2}-96 q^{3}-168 q^{4}-144 q^{5}-\cdots .
\end{aligned}
$$

The equality of the two summation would be obvious from the following:

$$
\begin{aligned}
\sum_{n=1}^{\infty} \frac{n q^{n}}{1-q^{n}} & =\sum_{n=1}^{\infty} \sum_{m=0}^{\infty} n q^{n(m+1)}=\sum_{n=1}^{\infty} \sum_{m=1}^{\infty} n q^{n m}, \\
\sum_{n=1}^{\infty} \frac{q^{n}}{\left(1-q^{n}\right)^{2}} & =\sum_{n=1}^{\infty}\left(\frac{q^{n}}{\left(1-q^{n}\right)} \frac{1}{\left(1-q^{n}\right)}\right)=\sum_{n=1}^{\infty}\left(\sum_{r=1}^{\infty} q^{r n}\right)\left(\sum_{s=0}^{\infty} q^{s n}\right) \\
& =\sum_{n=1}^{\infty}\left(\sum_{r=1}^{\infty} \sum_{s=0}^{\infty} q^{(r+s) n}\right)=\sum_{n=1}^{\infty} \sum_{t=1}^{\infty} t q^{t n} .
\end{aligned}
$$

The last step uses the fact for a fixed $t=r+s$; there are exactly $t$ pairs of $(r \geqslant 1, s \geqslant 0)$ satisfing it. $E_{2}$ is related to the Dedekind Eta function by $\frac{d}{d \tau} \ln \eta(\tau)=\frac{i \pi}{12} E_{2}(\tau)$.

\section{APPENDIX B: COMPARE TO LANGMANN'S EXPANSION}

E. Langmann developed an algorithm to derive the eigenvalue and eigenfunction of elliptic Calogero-Moser(-Sutherland) model for general particle number. ${ }^{14,15}$ In his paper, Ref. 14 , a series expansion for the 2-body eigenvalue when $q \ll 1$ is given(formulae 26(a)-26(d)). Although his method is different from the instanton and WKB methods we present in this paper, we will show that his expansion is equivalent to our expansion (34). Langmann considered the 2-particle Shrödinger equation,

$$
\left[-\left(\frac{\partial^{2}}{\partial x_{1}^{2}}+\frac{\partial^{2}}{\partial x_{2}^{2}}\right)+2 \lambda(\lambda-1) V\left(x_{1}-x_{2}\right)\right] \psi(\vec{x})=\mathcal{E} \psi(\vec{x}),
$$

with potential $V(x)=-\wp(x)+c_{0}$,

$$
\begin{aligned}
c_{0} & =\frac{1}{12}-2 \sum_{m=1}^{\infty} \frac{1}{\left(q^{m / 2}-q^{-m / 2}\right)^{2}}=\frac{1}{12} E_{2}(q) \\
& =\frac{1}{12}-2 q-6 q^{2}-8 q^{3}-14 q^{4}+\cdots
\end{aligned}
$$

After changing variables, equation becomes the Lamé equation (5) describing the relative motion of particles. The eigenvalue $\mathcal{E}$ is explicitly given as an expansion in $q: \mathcal{E}=\mathcal{E}_{0}+\mathcal{E}_{1} q+\mathcal{E}_{2} q^{2}+\mathcal{E}_{3} q^{3}$ $+\cdots$. Especially notice that, in the notation of paper, Ref. 14, the relation is

$$
2 \mathcal{E}_{0}=P^{2}+\left(n_{1}+n_{2}\right)^{2},
$$

where the term $\left(n_{1}+n_{2}\right)^{2}$ is the kinetic energy of center-of-mass motion.

The relation between the eigenvalues $\mathcal{E}$ and our $B$ is

$$
B=-2 \mathcal{E}+\left(n_{1}+n_{2}\right)^{2}+4 \lambda(\lambda-1) c_{0},
$$


provided we identify parameters as $P=v, \lambda=n$. Then, using relation (B4) to Langmann's expansion of $\mathcal{E}$, we can rewrite our expansion (34) in a slightly different form

$$
\begin{aligned}
B= & -v^{2}+\frac{n(n-1)}{3} E_{2}(q)-\frac{8 n^{2}(n-1)^{2} q}{v^{2}-1} \\
& -\frac{8 n^{2}(n-1)^{2} q^{2}}{\left(v^{2}-1\right)^{3}\left(v^{2}-4\right)} \times\left[n^{2}(n-1)^{2}\left(5 v^{2}+7\right)-12 n(n-1)\left(v^{2}-1\right)^{2}\right. \\
& \left.+6\left(v^{2}-1\right)^{2}\left(v^{2}-2\right)\right]+O\left(q^{3}\right) .
\end{aligned}
$$

Compare (34) and (B5), the difference between them is that we have collected some terms coming from instanton contribution in (34) which summed into $\frac{1}{3} n(n-1)\left(1-E_{2}\right)$, together with $-\frac{1}{3} n(n-1)\left(1-2 E_{2}(q)\right)$, which gives the term $\frac{1}{3} n(n-1) E_{2}(q)$ in (B5). This fact can be checked to higher order by calculating higher order instanton effects in gauge theory. Note that the value $\frac{1}{3} n(n-1)\left(1-E_{2}\right)$ exactly corresponds to the extra $\mathrm{U}(1)$ term contained in the prepotential of $\mathrm{U}(2)$ gauge theory, see Subsection IV B.

\section{APPENDIX C: A METHOD TO OBTAIN THE DIFFERENTIAL OPERATORS}

When we derive the differential operators $D_{n}\left(\omega, \partial_{\omega}, k\right)$, we need to separate from the integrand some terms of total derivative. By discarding these total derivative terms from the contour integral, we can simplify the differential operators as far as possible. It is necessary to find a systematic method to deal with higher order WKB perturbations to make the program complete. After some trial and error, we find a workable method.

The method is carried out as following.

In the first step, we solve the WKB relation for $p_{n}(\varkappa)$, explicitly carry out the derivatives, and the final expressions are of the form

$$
\begin{array}{ll}
p_{2 l+1}(\varkappa)=\frac{\operatorname{sn} \varkappa \operatorname{cn} \varkappa \mathrm{dn} \varkappa}{\left(\omega+\operatorname{sn}^{2} \varkappa\right)^{3 l+1}} \times\left(\text { polynomial of } \operatorname{sn}^{2} \varkappa\right), & l=0,1,2, \ldots, \\
p_{2 l}(\varkappa)=\frac{1}{\left(\omega+\operatorname{sn}^{2} \varkappa\right)^{3 l-\frac{1}{2}}} \times\left(\text { polynomial of } \operatorname{sn}^{2} \varkappa\right), & l=0,1,2, \ldots
\end{array}
$$

The $p_{2 l+1}(\varkappa)$ can be written as a total derivative term, so its contour integral is zero. $p_{1} \sim$ $\partial_{\varkappa}\left(\ln p_{0}\right)$ is special. The polynomials in the numerator of $p_{2 l+1}$ for $l \geqslant 1$ are of the form $c_{*} \operatorname{sn}^{6 l-2} \varkappa+$ $c_{*} \operatorname{sn}^{6 l-4} \varkappa+\cdots+c_{*} \operatorname{sn}^{2} \varkappa+c_{*}$, where we use $c_{*}$ to represent all coefficients whose explicit form is not important here; they are some polynomials of $k^{2}, \omega$. Keep in mind, in the following formulae, all $c_{*}$ (and later $a_{*}$ ) could be different from each other. Set $\omega+\operatorname{sn}^{2} \varkappa=t$, then $p_{2 l+1}$ can be written as

$$
p_{2 l+1}(\varkappa)=(\operatorname{sn} \varkappa \operatorname{cn} \varkappa \operatorname{dn} \varkappa)\left(c_{*} t^{-(3 l+1)}+c_{*} t^{-3 l}+c_{*} t^{-(3 l-1)}+\cdots c_{*} t^{-3}+c_{*} t^{-2}\right) .
$$

Every term in $(\mathrm{C} 3)$ is a total derivative given by

$$
c_{*} \partial_{\varkappa}\left(\omega+\mathrm{sn}^{2} \varkappa\right)^{-l^{\prime}}, \quad l^{\prime}=1,2, \ldots, 3 l ; \quad l \geqslant 1,
$$

with proper choice of the coefficient. Therefore, $\oint d \varkappa p_{2 l+1}(\varkappa)=0$ as expected.

So we only need to deal with $p_{2 l}$ for $l \geqslant 0$. The polynomials in the numerator of $p_{2 l}$ are of the form $c_{*} \mathrm{sn}^{6 l} \varkappa+c_{*} \operatorname{sn}^{6 l-2} \varkappa+\cdots+c_{*} \operatorname{sn}^{2} \varkappa+c_{*}$. Then $p_{2 l}$ can be expanded as a polynomial of $t$, let us denote it as $p_{2 l}^{(I)}$,

$$
p_{2 l}^{(I)}(\varkappa)=c_{*} t^{-\left(3 l-\frac{1}{2}\right)}+c_{*} t^{-\left(3 l-\frac{3}{2}\right)}+c_{*} t^{-\left(3 l-\frac{5}{2}\right)}+\cdots c_{*} t^{-\frac{1}{2}}+c_{*} t^{\frac{1}{2}} .
$$

It is generated by a differential operator acting on $p_{0} \sim \sqrt{t}$,

$$
\oint d \varkappa p_{2 l}^{(I)}(\varkappa)=\left(c_{*} \partial_{\omega}^{3 l}+c_{*} \partial_{\omega}^{3 l-1}+c_{*} \partial_{\omega}^{3 l-2}+\cdots c_{*} \partial_{\omega}+c_{*}\right) \oint d \varkappa p_{0}(\varkappa)
$$


The second step is to reduce the order of the differential operator for $p_{2 l}^{(I)}$. The method is to find proper total derivative terms like $\partial_{\varkappa}(* * *)$ that can substitute terms of $c_{*} t^{-\left(3 l-\frac{1}{2}\right)}+c_{*} t^{-\left(3 l-\frac{3}{2}\right)}+\cdots+$ $c_{*} t^{-\left(2 l+\frac{1}{2}\right)}$ in (C5), then abandon them in the contour integral $\oint p_{2 l}^{(I)} d x$. Therefore, the lowest order of polynomial integrand (C5) is increased and the corresponding differential operator for $p_{2 l}^{(I)}$ is also simplified. We find the total derivative terms are generated by

$$
c_{*} \partial_{\varkappa}^{2}\left(\omega+\operatorname{sn}^{2} \varkappa\right)^{-\left(3 l-\frac{3}{2}-l^{\prime}\right)}, \quad l^{\prime}=1,2, \ldots, 2 l-1 .
$$

A nice property of this term is that its final expression can be expressed in terms of $\operatorname{sn}^{2} \varkappa$, or in terms of $t$, as

$$
c_{*} t^{-\left(3 l+\frac{1}{2}-l^{\prime}\right)}+c_{*} t^{-\left(3 l-\frac{1}{2}-l^{\prime}\right)}+c_{*} t^{-\left(3 l-\frac{3}{2}-l^{\prime}\right)}+c_{*} t^{-\left(3 l-\frac{5}{2}-l^{\prime}\right)} .
$$

Now we can discard some terms in (C5) using (C7). For $l^{\prime}=1$, choosing proper coefficient in (C7), we can make the coefficient of the first term in (C8) equal to the coefficient of the first term in (C5); hence,

$$
\begin{aligned}
p_{2 l}^{(I)}(\varkappa)= & {\left[c_{*} \partial_{\varkappa}\left(\frac{\operatorname{sn} \varkappa \operatorname{cn} \varkappa \mathrm{dn} \varkappa}{\left(\omega+\operatorname{sn}^{2} \varkappa\right)^{3 l-\frac{3}{2}}}\right)-\left(c_{*} t^{-\left(3 l-\frac{3}{2}\right)}+c_{*} t^{-\left(3 l-\frac{5}{2}\right)}+c_{*} t^{-\left(3 l-\frac{7}{2}\right)}\right)\right] } \\
& +\left(c_{*} t^{-\left(3 l-\frac{3}{2}\right)}+c_{*} t^{-\left(3 l-\frac{5}{2}\right)}+\cdots c_{*} t^{-\frac{1}{2}}+c_{*} t^{\frac{1}{2}}\right) .
\end{aligned}
$$

Now the total derivative term can be abandoned, and the order of $p_{2 l}^{(I)}$ is increased to $t^{-\left(3 l-\frac{3}{2}\right)}$, meanwhile the coefficients of other three terms are changed. Repeat this process for $l^{\prime}=2$, we can increase the order of $p_{2 l}^{(I)}$ to $t^{-\left(3 l-\frac{5}{2}\right)}$, an so on. For every $l^{\prime} \in\{1,2, \ldots, l\}$, the process of removing (C7) is carried out once. After the process, we get the $p_{2 l}^{(I I)}$,

$$
p_{2 l}^{(I I)}(\varkappa)=c_{*} t^{-\left(2 l-\frac{1}{2}\right)}+c_{*} t^{-\left(2 l-\frac{3}{2}\right)}+c_{*} t^{-\left(2 l-\frac{5}{2}\right)}+\cdots c_{*} t^{-\frac{1}{2}}+c_{*} t^{\frac{1}{2}},
$$

and the associated differential operator,

$$
\oint d \varkappa p_{2 l}^{(I I)}(\varkappa)=\left(c_{*} \partial_{\omega}^{2 l}+c_{*} \partial_{\omega}^{2 l-1}+c_{*} \partial_{\omega}^{2 l-2}+\cdots c_{*} \partial_{\omega}+c_{*}\right) \oint d \varkappa p_{0}(\varkappa) .
$$

The third step is to minimize the differential operator. After carrying out the process for $l^{\prime}=1,2, \ldots, l$, we get $p_{2 l}^{(I I)}$ and the corresponding differential operator of the form in (C11). Remember that the coefficients $c_{*}$ in $(\mathrm{C} 11)$ are polynomials of $k^{2}, \omega$; it turns out that these coefficients can be further simplified. The reason is the following: we assume the differential operators for the Mathieu equation, conjectured in Ref. 33; take the simplest form we can get through the simplification process. We call these differential operators minimal. The differential operators of the Lamé equation, if they are minimal, should reduce to the minimal differential operators of the Mathieu equation in the limit $k \rightarrow 0, \omega \rightarrow(w-1) / 2$. However, we find the differential operator obtained after performing the second step for $l^{\prime}=1,2, \ldots, l$ is not minimal, some redundant terms can be further discarded. We should continue the process for $l^{\prime}=l+1, l+2, \ldots, 2 l-1$ to remove total derivative terms of (C7). For every $l^{\prime} \in\{l+1, l+2, \ldots, 2 l-1\}$, the process of removing (C7) may be repeated for a few times, depending on $l$. The simplification does not further decrease the order of the differential operator in (C11), but simplifies its coefficients to their minimal form.

Some details about this step would be helpful. Unlike the previous step where the whole coefficient $c_{*}$ is subtracted, the new issue here is that only some terms in the coefficients $c_{*}$ should be subtracted, and we have to determine which part. The key point here is that the criterion of "minimal" comes from results of the Mathieu equation; so in order to get a clue, in this step, we need to track the limit $k \rightarrow 0, \omega \rightarrow(w-1) / 2$ for the formulae we have obtained and compare them to the results of the Mathieu equation. In this limit, the $p_{2 l}^{(I I)}$ obtained from the second step becomes

$$
\begin{aligned}
\stackrel{\lim }{\longrightarrow} p_{2 l}^{(I I)}= & \left(\cdots+a_{*} w^{l-4}+a_{*} w^{l-2}+a_{*} w^{l}\right) \hat{t}^{-\left(2 l-\frac{1}{2}\right)} \\
& +\left(\cdots+a_{*} w^{l-5}+a_{*} w^{l-3}+a_{*} w^{l-1}\right) \hat{t}^{-\left(2 l-\frac{3}{2}\right)}+\cdots+a_{*} w \hat{t}^{-\left(l+\frac{1}{2}\right)}+a_{*} \hat{t}^{-\left(l-\frac{1}{2}\right)},
\end{aligned}
$$


with $a_{*}$ some numerical coefficients, the powers of $w$ are non-negative, and $\hat{t} \sim \sqrt{w-\cos 2 x}$ is the limit of $t$. From the results of the Mathieu equation, ${ }^{33}$ we know that the minimal form of the expression on the right hand side should be $a_{*} w^{l} \hat{t}^{-\left(2 l-\frac{1}{2}\right)}+a_{*} w^{l-1} \hat{t}^{-\left(2 l-\frac{3}{2}\right)}+\cdots+a_{*} w \hat{t}^{-\left(l+\frac{1}{2}\right)}+a_{*} \hat{t}^{-\left(l-\frac{1}{2}\right)}$; this indicates all other terms can be represented by total derivative terms and should be subtracted. It turns out that we should use formula (C7) with $l^{\prime}=l+1, l+2, \ldots, 2 l-1$. For example, in order to get rid of the term $a_{*} w^{l-2} \hat{t}^{-\left(2 l-\frac{1}{2}\right)}$ in (C12), we should subtract a term from $p_{2 l}^{(I I)}$ by

$$
p_{2 l}^{(I I)}-a_{*}(2 \omega+1)^{l-2} \partial_{\varkappa}^{2}\left(\omega+\operatorname{sn}^{2} \varkappa\right)^{-\left(3 l-\frac{3}{2}-(l+1)\right)},
$$

and the coefficient can be determined by comparing the coefficients of $w^{l-2} \hat{t}^{-\left(2 l-\frac{1}{2}\right)}$ in (C12) and in $\stackrel{\lim }{\longrightarrow}(2 \omega+1)^{l-2} \partial_{\chi}^{2}\left(\omega+\operatorname{sn}^{2} \varkappa\right)^{-\left(3 l-\frac{3}{2}-(l+1)\right)}$. Similarly, we can get rid of $w^{l-2 s} \hat{t}^{-\left(2 l-\frac{1}{2}\right)}$, with $s=$ $2,3, \ldots\left[\frac{l}{2}\right]$, by subtracting a term made of $(2 \omega+1)^{l-2 s} \partial_{\varkappa}^{2}\left(\omega+\operatorname{sn}^{2} \varkappa\right)^{-\left(3 l-\frac{3}{2}-(l+1)\right)}$. Formula (C7) with $l^{\prime}=l+1$ is repeatedly used for $\frac{l}{2}$ (for even $l$ ) or $\frac{l-1}{2}$ (for odd $l$ ) times. In the same way, we can get rid of the remaining unnecessary terms in the second non-minimal coefficient, $a_{*} w^{l-3} \hat{t}^{-\left(2 l-\frac{3}{2}\right)}, a_{*} w^{l-5}$ $\hat{t}^{-\left(2 l-\frac{3}{2}\right)}, \ldots$, etc.; formula (C7) with $l^{\prime}=l+2$ is repeatedly used for $\frac{l}{2}-1$ (for even $l$ ) or $\frac{l-1}{2}$ (for odd $l$ ) times. Continuing the process, we can minimize all coefficients in $(\mathrm{C} 12)$; in general, the coefficient of the term of order $\hat{t}^{-\left(2 l-\frac{1}{2}-r\right)}$ with $0 \leqslant r \leqslant l-2$ is minimized by repeatedly using (C7) with $l^{\prime}=l+1+r$ for $\frac{l-r}{2}$ (for even $l-r$ ) or $\frac{l-r-1}{2}$ (for odd $l-r$ ) times.

At last, we get the minimal polynomial $p_{2 l}^{(I I I)}$ and the associated minimal differential operator,

$$
\oint d \varkappa p_{2 l}^{(I I I)}(\varkappa)=\left(c_{*} \partial_{\omega}^{2 l}+c_{*} \partial_{\omega}^{2 l-1}+c_{*} \partial_{\omega}^{2 l-2}+\cdots c_{*} \partial_{\omega}+c_{*}\right) \oint d \varkappa p_{0}(\varkappa),
$$

now with $c_{*}$, still polynomials of $k^{2}, \omega$, be the minimal coefficients.

Using this method, we successfully derive differential operators for $p_{2 l}$ of the Lamé equation, for the first few $l$. In the limit $k \rightarrow 0, \omega \rightarrow(\omega-1) / 2$, these differential operators correctly reduce to the minimal differential operators of the Mathieu equation derived in Ref. 33.

Apparently, the minimal differential operators of the Lamé equation in form (64) can be obtained in the same way, with the total derivative terms for $p_{2 l}$ generated by

$$
c_{*} \partial_{\varkappa}^{2}\left(\widetilde{\omega}-\mathrm{cn}^{2} \varkappa\right)^{-\left(3 l-\frac{3}{2}-l^{\prime}\right)}, \quad l^{\prime}=1,2, \ldots, 2 l-1 .
$$

The relation between $D_{n}\left(\omega, \partial_{\omega}, k\right)$ and $\widetilde{D}_{n}\left(\widetilde{\omega}, \partial_{\widetilde{\omega}}, k\right)$ in (72) holds for $n \geqslant 2$. And the differential operators of the Mathieu equation can be obtained following the same steps, with the total derivative terms for $p_{2 l}$ generated by

$$
c_{*} \partial_{z}^{2}(w-\cos 2 z)^{-\left(3 l-\frac{3}{2}-l^{\prime}\right)}, \quad l^{\prime}=1,2, \ldots, 2 l-1,
$$

with the notation used in Ref. 33. This also gives an explanation for the conjectural form of $D_{n}\left(w, \partial_{w}\right)$ in that paper (in this paper denoted by $D_{n}\left(u, \partial_{u}\right)$ ).

${ }^{1}$ E. Mathieu, “Mémoire sur le mouvement vibratoire d'une membrane de forme elliptique,” J. Math. Pures Appl. 13, 137-203 (1868).

${ }^{2}$ G. Lamé, "Sur les surfaces isothermes dans les corps solides homogènes en équilibre de température," J. Math. Pures Appl. 2, 147-183 (1837).

${ }^{3}$ N. Seiberg and E. Witten, "Electric-magnetic duality, monopole condensation, and confinement in $\mathrm{N}=2$ supersymmetric Yang-Mills theory,” Nucl. Phys. B 426, 19-52 (1994).

${ }^{4}$ N. Seiberg and E. Witten, "Monopoles, duality and chiral symmetry breaking in N = 2 supersymmetric QCD," Nucl. Phys B 431, 484-550 (1994).

${ }^{5}$ N. Nekrasov and S. Shatashvili, "Supersymmetric vacum and Bethe ansatz," Nucl. Phys. B, Proc. Suppl. 192-193, 91-112 (2009).

${ }^{6}$ N. Nekrasov and S. Shatashvili, "Quantum integrability and supersymmetric vacua," Prog. Theor. Phys. Suppl. 177, 105 (2009).

${ }^{7}$ N. Nekrasov and S. Shatashvili, "Quantization of integrable systems and four dimensional gauge theories," in 16th International Congress on Mathematical Physics, 2009.

${ }^{8}$ S. S. Gubser and A. Hashimoto, "Exact absorption probabilities for the D3-brane," Commun. Math. Phys. 203, 325-340 (1999).

9 J. Lachapelle, "A study of the growth of entropy fluctuations during reheating in Roulette inflation," Master thesis, McGill University, 2008. 
${ }^{10}$ M. Abramowitz and I. A. Stegun, "Handbook of Mathematical Functions" (Dover Publications, Inc., New York, 1965).

${ }^{11}$ See http://dlmf.nist.gov/ for "NIST Digital Library of Mathematical Functions."

${ }^{12}$ H. J. W. Müller-Kirsten, Introduction to Quantum Mechanics: Schrödinger Equation and Path Integral (World Scientific, 2006).

${ }^{13}$ E. T. Whittaker and G. N. Watson, A Course of Modern Analysis (Cambridge University Press, 1927).

${ }^{14}$ E. Langmann, "An explicit solution of the (quantum) elliptic Calogero-Sutherland model," in Symmetry and Perturbation Theory (SPT04) (World Scientific, 2005).

${ }^{15}$ E. Langmann, "Explicit solution of the (quantum) elliptic Calogero-Sutherland model," Ann. Henri Poincare 15, 755-791 (2014).

${ }^{16}$ A. Gorsky, I. M. Krichever, A. Marshakov, A. Mironov, and A. Morozov, "Integrability and Seiberg-Witten exact solution," Phys. Lett. B 355, 466 (1995).

${ }^{17}$ E. Martinec and N. Warner, "Integrable systems and supersymmetric gauge theories," Nucl. Phys. B 459, 97-112 (1996).

${ }^{18}$ R. Donagi and E. Witten, "Supersymmetric Yang-Mills and integrable systems," Nucl. Phys. B 460, 299-334 (1996).

${ }^{19}$ H. Itoyama and A. Morozov, "Prepotential and the Seiberg-Witten theory," Nucl. Phys. B 491, 529-573 (1997).

${ }^{20}$ H. Itoyama and A. Morozov, "Integrability and Seiberg-Witten theory: Curves and periods," Nucl. Phys. B 477, 855-877 (1996).

${ }^{21}$ E. D' Hoker and D. H. Phong, "Lectures on supersymmetric Yang-Mills theory and integrable systems," e-print arXiv: hep-th/9912271.

${ }^{22}$ N. Nekrasov, "Seiberg-Witten prepotential from instanton counting," Adv. Theor. Math. Phys. 7, 831-864 (2004).

${ }^{23}$ N. Nekrasov and A. Okounkov, "Seiberg-Witten theory and random partitions," e-print arXiv:hep-th/0306238.

${ }^{24}$ G. W. Moore, N. Nekrasov, and S. Shatashvili, "Integrating over Higgs branches," Commun. Math. Phys. 209, 97 (2000).

${ }^{25}$ A. Losev, N. Nekrasov, and S. L. Shatashvili, "Issues in topological gauge theory," Nucl. Phys. B 534, 549 (1998).

${ }^{26}$ A. Losev, N. Nekrasov, and S. L. Shatashvili, "Testing Seiberg-Witten solution," in Strings, Branes and Dualities (Springer, Cargese, 1997), pp. 359-372.

${ }^{27}$ L. F. Alday and Y. Tachikawa, "Affine SL(2) conformal blocks from 4d gauge theories," Lett. Math. Phys. 94, 87-114 (2010).

${ }^{28}$ K. Maruyoshi and M. Taki, "Deformed prepotential, quantum integrable system and Liouville field theory," Nucl. Phys. B 841, 388-425 (2010).

${ }^{29}$ M. Matone, "Instantons and recursion relations in N = 2 Susy gauge theory," Phys. Lett. B 357, 342 (1995).

${ }^{30}$ R. Flume, F. Fucito, J. F. Morales, and R. Poghossian, “Matone's relation in the presence of gravitational couplings," J. High Energy Phys. 0404, 008 (2004).

${ }^{31}$ A. Mironov and A. Morozov, "Nekrasov functions and exact Bohr-Sommerfeld integrals," J. High Energy Phys. 04, 040 (2010).

${ }^{32}$ W. He and Y.-G. Miao, "Magnetic expansion of Nekrasov theory: The SU(2) pure gauge theory,” Phys. Rev. D 82, 025020 (2010).

${ }^{33}$ W. He and Y.-G. Miao, "Mathieu equation and elliptic curve," Commun. Theor. Phys. 58, 827-834 (2012).

${ }^{34}$ W. He, "Matone's relation of $\mathrm{N}=2$ super Yang-Mills and spectrum of Toda chain," Commun. Theor. Phys. 56, 905-912 (2011).

${ }^{35}$ R. Flume and R. Poghossian, "An algorithm for the microscopic evaluation of the coefficients of the Seiberg-Witten prepotential," Int. J. Mod. Phys. A 18, 2541 (2003).

${ }^{36}$ U. Bruzzo, F. Fucito, J. F. Morales, and A. Tanzini, “Multi-instanton calculus and equivariant cohomology,” J. High Energy Phys. 0305, 054 (2003).

${ }^{37}$ T. Okuda and V. Pestun, "On the instantons and the hypermultiplet mass of $\mathrm{N}=2^{*}$ super Yang-Mills on $\mathrm{S}^{4}$," J. High Energy Phys. 1203, 017 (2012).

${ }^{38}$ V. A. Fateev and A. V. Litvinov, “On AGT conjecture,” J. High Energy Phys. 1002, 014 (2010).

${ }^{39}$ I. S. Gradshteyn and I. M. Ryzhik, Table of Integrals, Series, and Products (Elsevier, 2007).

${ }^{40}$ P. Zinn-Justin, "Integrability and combinatorics: Selected topics," Lecture Notes for Les Houches (2008) (unpublished).

${ }^{41}$ N. Nekrasov, "Instanton partition functions and M-theory," Jpn. J. Math. 4, 63-93 (2009).

${ }^{42}$ L. F. Alday, D. Gaiotto, and Y. Tachikawa, "Liouville correlation functions from four dimensional gauge theories," Lett. Math. Phys. 91, 167 (2010).

${ }^{43}$ J. F. Wu, Y. Y. Xu, and M. Yu, "Recursions in Calogero-Sutherland model based on Virasoro singular vectors," Commun. Theor. Phys. 57, 743-758 (2012).

${ }^{44}$ Z.-X. Wang and D.-R. Guo, Special Functions (World Scientific, 1989).

45 C.-D. Pan and C.-B. Pan, Introduction to Modular Forms (Peking University Press, 2002).

46 J. V. Armitage and W. F. Eberlein, Elliptic Functions (Cambridge University Press, 2006).

${ }^{47}$ W. He, "A new treatment for some periodic Schrödinger operators," e-print arXiv:1412.6776. 